\title{
Universal Bounds for Size and Energy of Codes of Given Minimum and Maximum Distances
}

October 17, 2019

P. Boyvalenkov

peter@math.bas.bg

Institute for Mathematics and Informatics, BAS, Sofia 1113, Bulgaria

and Southwestern University, Blagoevgrad, Bulgaria

P. DRAGNEV

dragnevp@pfw.edu

Department of Mathematical Sciences, PFW, Fort Wayne, IN 46805, USA

D. HARDIN

doug.hardin@vanderbilt.edu

Department of Mathematics, Vanderbilt University, Nashville, TN, 37240, USA

E. SAFF

edward.b.saff@vanderbilt.edu

Department of Mathematics, Vanderbilt University, Nashville, TN, 37240, USA

M. STOYANOVA

stoyanova@fmi.uni-sofia.bg

Faculty of Mathematics and Informatics, Sofia University "St. Kliment Ohridski", Sofia 1164, Bulgaria

\begin{abstract}
We employ signed measures that are positive definite up to certain degrees to establish Levenshtein-type upper bounds on the cardinality of codes with given minimum and maximum distances, and universal lower bounds on the potential energy (for absolutely monotone interactions) for codes with given maximum distance and cardinality. The distance distributions of codes that attain the bounds are found in terms of the parameters of Levenshtein-type quadrature formulas. Necessary and sufficient conditions for the optimality of our bounds are derived. Further, we obtain upper bounds on the energy of codes of fixed minimum and maximum distances and cardinality.
\end{abstract}

Keywords - bounds for codes, linear programming, energy of codes.

\section{Introduction}

Let $F_{q}$ be an alphabet of size $q$. We consider codes (sets) $C \subset F_{q}^{n}=\left\{\left(x_{1}, \ldots, x_{n}\right): x_{i} \in F_{q}\right\}$ with the Hamming distance $d(x, y)$ between words $x, y \in F_{q}^{n}$. In setting of $F_{q}^{n}$ as a polynomial metric space [26] the following change of the variable

$$
t=1-\frac{2 d}{n} \in T_{n}:=\left\{t_{i}=-1+\frac{2 i}{n}: i=0,1, \ldots, n\right\}
$$


is very convenient. It brings the distances to "inner" products and for $x, y \in F_{q}^{n}$ we write

$$
\langle x, y\rangle=1-\frac{2 d(x, y)}{n}=t_{n-d} \in T_{n}
$$

For any code $C \subset F_{q}^{n}$ we use

$$
\begin{aligned}
& s(C):=\max \{\langle x, y\rangle: x, y \in C, x \neq y\} \in T_{n}, \\
& \ell(C):=\min \{\langle x, y\rangle: x, y \in C, x \neq y\} \in T_{n},
\end{aligned}
$$

to denote the counterparts of the minimum and maximum distance of $C$, respectively. Denote by

$$
C_{n, q}(\ell, s):=\left\{C \subset F_{q}^{n} \mid s(C) \leq s, \ell(C) \geq \ell\right\}
$$

the set of codes in $F_{q}^{n}$ with pairwise distances greater than or equal to the minimum distance $d:=n(1-s) / 2$ and less than or equal to the maximum distance $D:=n(1-\ell) / 2$. Let

$$
\mathcal{A}_{q}(n, \ell, s):=\max \left\{|C|: C \in C_{n, q}(\ell, s)\right\}
$$

be the maximum possible cardinality of a code from $C_{n, q}(\ell, s)$. The investigation of the quantities like $\mathcal{A}_{q}(n, \ell, s)$ is one of the classical problems in the coding theory.

We are interested also in a minimum energy problem which is somewhat more general but turns out to be closely related.

Definition 1.1 Given a (potential) function $h(t):[-1,1] \rightarrow[0,+\infty]$ and a code $C \subset F_{q}^{n}$, the potential energy (also referred to as $h$-energy) of $C$ is

$$
E_{h}(C):=\sum_{x, y \in C, x \neq y} h(\langle x, y\rangle) .
$$

While we only need the values of $h$ on the discrete set $T_{n}$ for computing the $h$-energy, we further assume that $h$ is (strictly) absolutely monotone on the interval $[-1,1)$; that is, $h$ and all its derivatives are defined and (positive) nonnegative on this interval. This approach facilitates our investigation and the explanation of our results. We remark that the function $F(z)=h(t)$, where $z=n(1-t) / 2$, is completely monotone on $(0, n]$ (i.e., $(-1)^{k} F^{(k)}(z) \geq 0$ for all $\left.z \in(0, n]\right)$ if and only if $h$ is absolutely monotone on $[-1,1]$.

For absolutely monotone potentials $h$ we consider the quantity

$$
\mathcal{E}_{h}(n, M, \ell):=\min \left\{E_{h}(C): C \in C_{n, q}(\ell, 1-2 / n),|C|=M\right\},
$$

the smallest possible $h$-energy of a code from $C_{n, q}(\ell, 1-2 / n)$ with prescribed $M$.

General linear programming bounds for quantities like $\mathcal{A}_{q}(n, \ell, s)$ and $\mathcal{E}_{h}(n, M, \ell)$ were first introduced by Delsarte [17] (see [18,26] and references therein) and Yudin [29]. Linear programming bounds for energies of codes and designs in different spaces (including $F_{q}^{n}$ ) were investigated for the first time by Ashikhmin-Barg [1, Ashikhmin-Barg-Litsyn [2] (see also [3, 4] Energies of codes in $F_{q}^{n}$ were considered in 2014 by Cohn and Zhao [15] (see also [14]) with a focus on (universally) optimal codes and by the authors [9] who focused on universal bounds. 
In this paper we use linear programming techniques to derive explicit upper bounds for $\mathcal{A}_{q}(n, \ell, s)$ and lower bounds for $\mathcal{E}_{h}(n, M, \ell)$. Our bounds can be computed for all feasible values of $q, n, s$, and $\ell$, which makes them universal in the sense of Levenshtein [26]. We are not aware of such explicit universal bounds in the existing literature (see [21] for a particular case) more than 20 years after the chapter [26] by Levenshtein and the paper [18] by Delsarte and Levenshtein.

There is an intricate interplay between the Levenshtein universal bounds for $\mathcal{A}_{q}(n,-1, s)$ and universal lower bounds on $\mathcal{E}_{h}(n, M,-1)$ in different polynomial metric spaces (see 8 for Euclidean spheres $\mathbb{S}^{n-1}$ and [9] for Hamming spaces $F_{q}^{n}$ ). We further that relationship to corresponding bounds for codes from $C_{n, q}(\ell, 1-2 / n)$ to derive and investigate simultaneously our cardinality and energy bounds.

For any real polynomial $f(t)$ we consider its expansion in Krawtchouk polynomials (see Section 2),

$$
f(t)=\sum_{i=0}^{n} f_{i} Q_{i}^{(n, q)}(t)
$$

(if the degree of the polynomial $f(t)$ exceeds $n$, then $f(t)$ is taken modulo $\prod_{i=0}^{n}\left(t-t_{i}\right)$ ) and set

$$
F_{\geq}:=\left\{f(t): f_{0}>0, f_{i} \geq 0, i=1,2, \ldots, n\right\} .
$$

If $f_{i}>0$ for $i=0,1,2, \ldots, \operatorname{deg}(f)$, then we write $f(t) \in F_{>}$. The coefficient $f_{0}$ is of special interest and we call it the zeroth coefficient of $f(t)$.

Following Delsarte [17], we have

$$
\mathcal{A}_{q}(n, \ell, s) \leq \min _{f \in \mathcal{F}_{n, \ell, s}} \frac{f(1)}{f_{0}}
$$

where

$$
\mathcal{F}_{n, \ell, s}:=\left\{f \in F_{\geq}: f(t) \leq 0, t \in[\ell, s]\right\} .
$$

Similarly, following Yudin [29], we have

$$
\mathcal{E}_{h}(n, M, \ell) \geq \max _{g \in \mathcal{G}_{n, \ell}^{(h)}} M\left(M g_{0}-g(1)\right)
$$

where

$$
\mathcal{G}_{n, \ell}^{(h)}:=\left\{g \in F_{\geq}: g(t) \leq h(t), t \in[\ell, 1)\right\} .
$$

Therefore, major results in this context crucially depend on proper choice and investigation of polynomials that optimize (2) or (3).

The Levenshtein bound (see [24, 26]) and the energy bound [9] work for $\ell=-1$ and, of course, depend on the properties of Krawtchouk polynomials and their adjacent polynomials which are orthogonal with respect to classical positive measures. The case $\ell>-1$, however, already involves more challenging signed measures. In this paper we develop the necessary theory of signed measures to be used in the investigation of the optimization problems arising from the right hand sides of (2) and (3). Then we derive and investigate universal upper bounds for $\mathcal{A}_{q}(n, \ell, s)$ and lower bounds for $\mathcal{E}_{h}(n, M, \ell)$. 
The paper is organized as follows. In Sections 2 and 3 we introduce the so-called adjacent polynomials and signed measures. Then we establish the positive definiteness of the corresponding measures up to appropriate degrees. Properties of the associated orthogonal (and adjacent again) polynomials are derived and discussed in Section 4 , where we define Levenshtein-type polynomials $f_{2 k}^{n, \ell, s}(t)$ to be used in (2). A Levenshtein-type quadrature formula is derived with nodes the roots of $f_{2 k}^{n, \ell, s}(t)$ to serve in proofs and properties. In Section 5 we obtain simultaneously Levenshteintype upper bounds on $\mathcal{A}_{q}(n, \ell, s)$ and (as in the case $\ell=-1$ ) the strongly related lower bounds on $\mathcal{E}_{h}(n, M, \ell)$. An important role in the proof is played by what we call the $(k, \ell)$-strengthened Krein condition extending the Levenshtein's strengthened Krein condition. Section 6 is devoted to description of codes which would attain our bounds. The distance distributions of such codes are found as functions of corresponding quadrature formulas parameters. In Section 7 we prove necessary and sufficient conditions for the optimality of our bounds (in other words, for existence of improving polynomials from the sets $\mathcal{F}_{n, \ell, s}$ and $\mathcal{G}_{n, \ell}^{(h)}$ ). The optimality (or existence of improvements) happens only simultaneously for both bounds. A linear programming refinement of our bounds is discussed in Section 8, where we provide evidence that in most cases the the nodes of our polynomials serve as the best approximation for the general linear programming solution. Upper bounds on the energy of the codes from $C_{n, q}(\ell, s)$ (including the case $\ell=-1$ ) of fixed cardinality $M$ are derived in Section 9 providing this way a strip where the energies of all such codes belong. Examples are shown in Section 10 where we build a Levenshtein-type system of bounds for a fixed $\ell$.

\section{Krawtchouk and adjacent polynomials}

For fixed $n$ and $q$, the (normalized) Krawtchouk polynomials are defined by

$$
Q_{i}^{(n, q)}(t):=\frac{1}{r_{i}} K_{i}^{(n, q)}(z)
$$

where

$$
z=\frac{n(1-t)}{2}
$$

is a change of the variable between the set $\{0,1, \ldots, n\}$ of the distances in $F_{q}^{n}$ and the set $T_{n}$,

$$
r_{i}:=(q-1)^{i}\left(\begin{array}{c}
n \\
i
\end{array}\right), i=0,1, \ldots, n
$$

are certain dimensions of spaces of functions (see also (5) below), and

$$
K_{i}^{(n, q)}(z):=\sum_{j=0}^{i}(-1)^{j}(q-1)^{i-j}\left(\begin{array}{c}
z \\
j
\end{array}\right)\left(\begin{array}{c}
n-z \\
i-j
\end{array}\right),
$$

$i=0,1, \ldots, n$, are the (usual) Krawtchouk polynomials corresponding to $F_{q}^{n}$ (see [28, Section $2.82])$. In the sequel we will omit the index $(n, q)$ in the notation of Krawtchouk polynomials. 
The polynomials $\left\{Q_{i}(t)\right\}_{i=0}^{n}$ form a basis of the space $\mathcal{P}_{n}$ of real polynomials of degree at most $n$ and satisfy the following three-term recurrence relation

$$
\left(t-a_{i}\right) Q_{i}(t)=b_{i} Q_{i+1}(t)+c_{i} Q_{i-1}(t)
$$

$i=1,2, \ldots, n-1$, with initial conditions $Q_{0}(t)=1$ and $Q_{1}(t)=(q t+q-2) /(2(q-1))$, where

$$
\begin{gathered}
a_{i}=-\frac{(q-2)(n-2 i)}{q n}, \\
b_{i}=\frac{2(q-1)(n-i)}{q n}, \quad c_{i}=\frac{2 i}{q n} .
\end{gathered}
$$

The measure of orthogonality for the system $\left\{Q_{i}(t)\right\}_{i=0}^{n}$ is discrete and given by

$$
\mu_{n}:=q^{-n} \sum_{i=0}^{n} r_{n-i} \delta_{t_{i}},
$$

where $\delta_{t_{i}}$ is the Dirac-delta measure at $t_{i} \in T_{n}$. The form

$$
\langle f, g\rangle=\int f(t) g(t) d \mu_{n}(t)
$$

defines an inner product over the class of polynomials of degree at most $n$. Note that

$$
r_{i}=\left(\int_{-1}^{1}\left(Q_{i}(t)\right)^{2} d \mu_{n}(t)\right)^{-1}=\left\|Q_{i}\right\|^{-2}
$$

We also need $(1,0)$ and $(1,1)$ adjacent polynomials as introduced by Levenshtein (cf. [26, Section 6.2], see also [24,25]). Denote

$$
T_{i}(x, y):=\sum_{j=0}^{i} r_{j} Q_{j}(x) Q_{j}(y),
$$

and define [26, Eq. (5.65)]

$$
Q_{i}^{1,0}(t):=\frac{T_{i}(t, 1)}{T_{i}(1,1)}, \quad i=0,1, \ldots, n-1 .
$$

Similarly, denote

$$
T_{i}^{1,0}(x, y):=\sum_{j=0}^{i} r_{j}^{1,0} Q_{j}^{1,0}(x) Q_{j}^{1,0}(y)
$$

where

$$
r_{j}^{1,0}=\frac{\left(\sum_{u=0}^{j} r_{u}\right)^{2}}{\left(\begin{array}{c}
n-1 \\
j
\end{array}\right)(q-1)^{j}}, j=0,1, \ldots, n-1,
$$


are the $(1,0)$ counterparts of $r_{j}$, and define [26, Eq. (5.68)]

$$
Q_{i}^{1,1}(t):=\frac{T_{i}^{1,0}(t,-1)}{T_{i}^{1,0}(1,-1)}, \quad i=0,1, \ldots, n-2 .
$$

Note that

$$
r_{j}^{1,1}=\frac{\left(\sum_{u=0}^{j}\left(\begin{array}{c}
n-1 \\
u
\end{array}\right)(q-1)^{u}\right)^{2}}{\left(\begin{array}{c}
n-2 \\
j
\end{array}\right)(q-1)^{j}}, j=0,1, \ldots, n-2,
$$

give the explicit formulas for the $(1,1)$ norm of the polynomials $Q_{j}^{1,1}(t)$ similatly to (5)).

The corresponding measures of orthogonality of the systems $\left\{Q_{i}^{1,0}(t)\right\}_{i=0}^{n-1}$ and $\left\{Q_{i}^{1,1}(t)\right\}_{i=0}^{n-2}$ are

$$
c^{1,0}(1-t) d \mu_{n}(t), \quad c^{1,1}(1-t)(1+t) d \mu_{n}(t)
$$

respectively, where

$$
c^{1,0}=\frac{q}{2(q-1)}, \quad c^{1,1}=\frac{n q^{2}}{4(n-1)(q-1)}
$$

are normalizing constants (see [26, Section 6.2]). Of course, the adjacent polynomials also satisfy corresponding three-term recurrence relations

$$
\left(t-a_{i}^{1, \varepsilon}\right) Q_{i}^{1, \varepsilon}(t)=b_{i}^{1, \varepsilon} Q_{i+1}^{1, \ell}(t)+c_{i}^{1, \varepsilon} Q_{i-1}^{1, \varepsilon}(t),
$$

where $\varepsilon \in\{0,1\}, b_{i}^{1, \varepsilon}>0$ is the ratio of the leading coefficients of $Q_{i+1}^{1, \varepsilon}(t)$ and $Q_{i}^{1, \varepsilon}(t), c_{i}^{1, \varepsilon}=$ $r_{i-1}^{1, \varepsilon} b_{i-1}^{1, \varepsilon} / r_{i}^{1, \varepsilon}>0$ and $a_{i}^{1, \varepsilon}=1-b_{i}^{1, \varepsilon}-c_{i}^{1, \varepsilon}$.

Note also the explicit relations [25]

$$
\begin{gathered}
Q_{i}^{1,0}(t)=\frac{K_{i}^{(n-1, q)}(z-1)}{\sum_{j=0}^{i} r_{j}}, \\
Q_{i}^{1,1}(t)=\frac{K_{i}^{(n-2, q)}(z-1)}{\sum_{j=0}^{i}\left(\begin{array}{c}
n-1 \\
j
\end{array}\right)(q-1)^{j}},
\end{gathered}
$$

where $z=n(1-t) / 2$ as above, between the $(1,0)$ and $(1,1)$ adjacent polynomials and the usual Krawtchouk polynomials.

For $\ell \in T_{n}$ we shall introduce below further adjacent polynomials $Q_{i}^{1, \ell}(t)$ as generalizations of $Q_{i}^{1,1}(t)$ (note that $\ell=-1$ in $Q_{i}^{1, \ell}(t)$ gives $Q_{i}^{1,1}(t)$ by the definitions in [26, Eqn. (5.66)] and (21) below). Under certain natural conditions the polynomials $Q_{i}^{1, \ell}(t)$ are orthogonal with respect to a signed measure $d \mu_{n, \ell}(t)$ which is defined and investigated below. With the next step, we shall use this new series to construct polynomials $Q_{i}^{1, \ell, s}(t)$ which are orthogonal with respect to another signed measure $d \mu_{n, \ell, s}(t)$ again to be defined and investigated below. Furthermore, the signed measures $d \mu_{n, \ell}(t)$ and $d \mu_{n, \ell, s}(t)$ are strong enough to imply properties which are crucial for our constructions. Then our Levenshtein-type polynomials will be constructed to be applied in (2) and, moreover, as in the case $\ell=-1$ [9], to setup polynomials to be applied in (3). In all 
these constructions and investigations, the Christoffel-Darboux formula [28, Chapter 3.2] plays an important role.

These Levenshtein-type polynomials can also be viewed as adjacent polynomials summarized by the following sequence:

$$
Q_{i} \rightarrow Q_{i}^{1,0} \rightarrow Q_{i}^{1, \ell} \rightarrow Q_{i}^{1, \ell, s}
$$

where each subsequent family of polynomials can be expressed in terms of the previous family using the Christoffel-Darboux formula (see (17), (91), (21), and (25)).

We conclude this section with notations for the zeros of the polynomials from the sequence (11). Let

$$
t_{i, 1}^{a}<t_{i, 2}^{a}<\cdots<t_{i, i}^{a}
$$

be the zeros of the polynomial $Q_{i}^{a}(t), i=0,1, \ldots$, , where the index $a$ stands for the pairs $(1,0)$, $(1,1),(1, \ell)$, or the triple $(1, \ell, s)$, respectively.

\section{Positive definite signed measures}

Signed measures were first used by Cohn and Kumar in [14 in the context of linear programming bounds for energy of spherical codes.

Definition 3.1 A signed Borel measure $\mu$ on $\mathbb{R}$ for which all polynomials are integrable is called positive definite up to degree $m$ if for all real polynomials $p \not \equiv 0$ of degree at most $m$ we have $\int p^{2}(t) d \mu(t)>0$. For such $\mu$, the bi-linear form

$$
\langle f, g\rangle_{\mu}:=\int f(t) g(t) d \mu(t)
$$

is an inner product on the space $\mathcal{P}_{m}$.

Suppose that $k, \ell$, and $s$ are such that the roots of $Q_{k}^{1,0}(t)$ lie in the open interval $(\ell, s) \subset$ $(-1,1)$; that is, $($ note $\ell<0)$

$$
-1 \leq \ell<t_{k, 1}^{1,0}<t_{k, k}^{1,0}<s \leq 1 .
$$

Then we define the following signed measures on $[-1,1]$ (see (4) and (10))

$$
\begin{aligned}
d \mu_{n, \ell}(t) & :=c^{1, \ell}(t-\ell)(1-t) d \mu_{n}(t), \\
d \mu_{n, s}(t) & :=c^{1, s}(s-t)(1-t) d \mu_{n}(t), \\
d \mu_{n, \ell, s}(t) & :=c^{1, \ell, s}(t-\ell)(s-t)(1-t) d \mu_{n}(t) .
\end{aligned}
$$

The normalizing constants in (14)-(16) are given by

$$
\begin{aligned}
c^{1, \ell} & :=\frac{n q^{2}}{2(q-1)(2(n-1)-n q(1+\ell))}, \\
c^{1, s} & :=\frac{n q^{2}}{2(q-1)[n q(1+s)-2(n-1)]},
\end{aligned}
$$




$$
c^{1, \ell, s}:=\frac{n^{2} q^{3}}{\left.2(q-1)\left[4(n-1)\left(n q d_{1}-n-q+2\right)-n^{2} q^{2} d_{2}\right)\right]},
$$

where $d_{1}=(2+\ell+s) / 2$ and $d_{2}=(1+\ell)(1+s)$. We will show below that for $n, q, \ell$ and $s$ that satisfy (13) the constants $c^{1, \ell}, c^{1, s}$, and $c^{1, \ell, s}$ are all positive.

The following theorem establishes the positive definiteness of the signed measures (14)-(16) up to degrees $k-1, k-1$, and $k-2$, respectively, as a consequence of the appropriate location (13) of $\ell$ and $s$.

Theorem 3.2 For given positive integers $n \geq 2$ and $q \geq 2$, let $k$, $\ell$, and $s$ satisfy the inequalities (13). Then the measures $\mu_{n, \ell}, \mu_{n, s}$ and $\mu_{n, \ell, s}$ are positive definite up to degrees $k-1, k-1$, and $k-2$, respectively.

Proof. Modifying the classical Radau quadrature [16, Sec. 2.7] for integration with respect to discrete measures we conclude that the zeros of the corresponding discrete orthogonal polynomial, the system of $k+1$ nodes

$$
t_{k, 1}^{1,0}<t_{k, 2}^{1,0}<\cdots<t_{k, k}^{1,0}<1
$$

defines a positive (i.e., the weights $w_{i}, i=1,2, \ldots, k+1$, are positive) Radau quadrature with respect to $\mu_{n}$,

$$
f_{0}:=\int_{-1}^{1} f(t) d \mu_{n}(t)=w_{k+1} f(1)+\sum_{i=1}^{k} w_{i} f\left(t_{k, i}^{1,0}\right),
$$

that is exact for all polynomials of degree at most $2 k$.

Using (17) for $f(t)=(t-\ell)(1-t)$, we find that

$$
\left(c^{1, \ell}\right)^{-1}=\sum_{i=1}^{k} w_{i}\left(t_{k, i}^{1,0}-\ell\right)\left(1-t_{k, i}^{1,0}\right)>0 .
$$

Similarly, we can show that $c^{1, s}>0$ and $c^{1, \ell, s}>0$.

Next, we apply (17) for $q(t)$, an arbitrary polynomial of degree at most $k-1$, to see that

$$
\begin{aligned}
& \int_{-1}^{1} q^{2}(t) d \mu_{n, \ell}(t) \\
= & c^{1, \ell} \int_{-1}^{1} q^{2}(t)(t-\ell)(1-t) d \mu_{n}(t) \\
= & c^{1, \ell} \sum_{i=1}^{k} w_{i} q^{2}\left(t_{k, i}^{1,0}\right)\left(t_{k, i}^{1,0}-\ell\right)\left(1-t_{k, i}^{1,0}\right) \geq 0 .
\end{aligned}
$$

The equality holds only if $q\left(t_{k, i}^{1,0}\right)=0$ for all $i=1, \ldots, k$, which would imply that $q(t) \equiv 0$. Therefore the measure $d \mu_{n, \ell}(t)$ is positive definite up to degree $k-1$. That $\mu_{n, s}$ is positive definite up to degree $k-1$ provided $s>t_{k, k}^{1,0}$ follows similarly. 
Finally, if $q(t) \not \equiv 0$ has degree at most $k-2$, then we utilize (17) again to see that

$$
\begin{aligned}
& \int_{-1}^{1} q^{2}(t) d \mu_{n, \ell, s}(t) \\
= & c^{1, \ell, s} \int_{-1}^{1} q^{2}(t)(t-\ell)(s-t)(1-t) d \mu_{n}(t) \\
= & c^{1, \ell, s} \sum_{i=1}^{k} w_{i} q^{2}\left(t_{k, i}^{1,0}\right)\left(t_{k, i}^{1,0}-\ell\right)\left(s-t_{k}^{1,0}\right)\left(1-t_{k, i}^{1,0}\right)>0 .
\end{aligned}
$$

This implies that the measure $d \mu_{n, \ell, s}(t)$ is positive definite up to degree $k-2$, as required.

Theorem 3.2 allows us to define orthogonal polynomials with respect to the corresponding signed measures. This provides essential ingredients for modifying Levenshtein's framework.

\section{Construction of Levenshtein-type polynomials}

\subsection{Existence and uniqueness of $Q_{j}^{1, \ell}(t), j=0,1, \ldots, k$, and $Q_{j}^{1, \ell, s}(t), j=0,1, \ldots, k-$ 1}

Some of the basic properties of orthogonal polynomials are no longer valid for series of polynomials generated by signed measures. Fortunately, our measures $d \mu_{n, \ell}(t)$ and $d \mu_{n, \ell, s}(t)$ possess the necessary properties by Theorem 3.2. Applying Gram-Schmidt orthogonalization we derive the existence and uniqueness (for the so-chosen normalizations) of the following two classes of orthogonal polynomials thus completing the sequence (11).

Theorem 4.1 For given positive integers $n \geq 2, q \geq 2$, let $k$, $\ell$, and $s$ satisfy the inequalities (13). The following two classes of orthogonal polynomials are well-defined:

$$
\begin{gathered}
\left\{Q_{j}^{1, \ell}(t)\right\}_{j=0}^{k} \text {, w.r.t. } d \mu_{n, \ell}(t), Q_{j}^{1, \ell}(1)=1 ; \\
\left\{Q_{j}^{1, \ell, s}(t)\right\}_{j=0}^{k-1}, \text { w.r.t. } d \mu_{n, \ell, s}(t), Q_{j}^{1, \ell, s}(1)=1 .
\end{gathered}
$$

The polynomials in both classes satisfy a three-term recurrence relation and their zeros interlace.

For our purposes we shall restrict to values of $\ell$ such that

$$
\frac{Q_{k+1}^{1,0}(\ell)}{Q_{k}^{1,0}(\ell)}<1 .
$$

As shown in the proof of Theorem 4.2 below the condition (18) is equivalent to the requirement for the largest zero of $Q_{k}^{1, \ell}(t)$ to be less than 1 . 


\subsection{Explicit construction and investigation of the polynomials $Q_{j}^{1, \ell}(t), j=$ $0,1, \ldots, k$}

The explicit form of the polynomials $Q_{j}^{1, \ell}(t)$ can be seen as a straightforward generalization of (6) (17) by using $\ell$ instead of -1 . We utilize the Christoffel-Darboux formula (see, for example [28, Th. 3.2.2], [26, Eq. (5.65)])

$$
\frac{T_{i}^{1,0}(x, y)}{r_{i}^{1,0} b_{i}^{1,0}}=\frac{Q_{i+1}^{1,0}(x) Q_{i}^{1,0}(y)-Q_{i+1}^{1,0}(y) Q_{i}^{1,0}(x)}{x-y}
$$

(when $x=y$ appropriate derivatives are used) in our construction. Moreover, similarly to [26], we use (19) to prove the interlacing properties of the zeros of $\left\{Q_{i}^{1, \ell}(t)\right\}$ with respect to the zeros of $\left\{Q_{i}^{1,0}(t)\right\}$.

In what follows in this and the next sections we assume that

$$
t_{k+1,1}^{1,0}<\ell<t_{k, 1}^{1,0}
$$

Theorem 4.2 Let $n, q, k$, and $\ell$ be such that (18) and (20) are satisfied. Then

$$
Q_{i}^{1, \ell}(t)=\frac{T_{i}^{1,0}(t, \ell)}{T_{i}^{1,0}(1, \ell)}=\eta_{i}^{1, \ell} t^{i}+\cdots, \quad i=0,1, \ldots, k,
$$

with $\eta_{i}^{1, \ell}>0$ and the polynomial $Q_{i}^{1, \ell}(t)$ has $i$ simple zeros $t_{i, 1}^{1, \ell}<t_{i, 2}^{1, \ell}<\cdots<t_{i, i}^{1, \ell}$ in the interval $(\ell, 1)$. Furthermore, the following interlacing properties

$$
\begin{aligned}
& t_{i, j}^{1, \ell} \in\left(t_{i, j}^{1,0}, t_{i+1, j+1}^{1,0}\right), i=1, \ldots, k-1, j=1, \ldots, i ; \\
& t_{k, j}^{1, \ell} \in\left(t_{k+1, j+1}^{1,0}, t_{k, j+1}^{1,0}\right), j=1, \ldots, k-1,
\end{aligned}
$$

and, finally, $t_{k, k}^{1, \ell} \in\left(t_{k+1, k+1}^{1,0}, 1\right)$ hold true.

Proof. It follows from (19) that the kernel $T_{i}^{1,0}(t, \ell)$ is orthogonal to any polynomial of degree at most $i-1$ with respect to the measure $\mu_{n, \ell}(t)$. Hence (21) follows from the positive definiteness of $d \mu_{n, \ell}(t)$ up to degree $k-1$ and the uniqueness of the Gram-Schmidt orthogonalization process (note also the normalization). The comparison of coefficients in (21) yields $\eta_{i}^{1, \ell}>0, i=0,1, \ldots, k$.

Next, it follows from (19) and (21) that the solutions of the equation

$$
\frac{Q_{i+1}^{1,0}(t)}{Q_{i}^{1,0}(t)}=\frac{Q_{i+1}^{1,0}(\ell)}{Q_{i}^{1,0}(\ell)}
$$

are the zeros of $Q_{i}^{1, \ell}(t)$ and the number $\ell$.

For every $i<k$ the zeros of $Q_{i+1}^{1,0}(t)$ and $Q_{i}^{1,0}(t)$ are interlaced and contained in the interval $\left[t_{k, 1}^{1,0}, t_{k, k}^{1,0}\right]$. Since $\operatorname{sign} Q_{i}^{1,0}(\ell)=(-1)^{i}$, we have $Q_{i+1}^{1,0}(\ell) / Q_{i}^{1,0}(\ell)<0$. The rational function $Q_{i+1}^{1,0}(t) / Q_{i}^{1,0}(t)$ has simple poles at $t_{i, j}^{1,0}, j=1, \ldots, i$, and simple zeros at $t_{i+1, j}^{1,0}, j=1, \ldots, i+1$. 
Therefore, there is at least one solution $t_{i, j}^{1, \ell}$ of (23) on each interval $\left(t_{i, j}^{1,0}, t_{i+1, j+1}^{1,0}\right), j=1, \ldots, i$, which accounts exactly for the zeros of $Q_{i}^{1, \ell}(t)$.

When $i=k$ we have $Q_{k+1}^{1,0}(\ell) / Q_{k}^{1,0}(\ell)>0$. Since $\ell \in\left(t_{k+1,1}^{1,0}, t_{k, 1}^{1,0}\right)$, we account similarly for the first $k-1$ solutions of (23), namely $t_{k, j}^{1, \ell} \in\left(t_{k+1, j+1}^{1,0}, t_{k, j+1}^{1,0}\right), j=1, \ldots, k-1$, to establish the interlacing properties (22). For the last zero of $Q_{k}^{1, \ell}(t)$ we use the fact that $Q_{k+1}^{1,0}(t) / Q_{k}^{1,0}(t)>0$ for $t \in\left(t_{k+1, k+1}^{1,0}, \infty\right)$. As $\lim _{t \rightarrow \infty} Q_{k+1}^{1,0}(t) / Q_{k}^{1,0}(t)=\infty$, we have one more solution $t_{k, k}^{1, \ell}>t_{k+1, k+1}^{1,0}$ of (23). Then (18) implies that $t_{k, k}^{1, \ell}<1$ because $Q_{k+1}^{1,0}(1) / Q_{k}^{1,0}(1)=1$.

The positive definiteness of the measure $d \mu_{n, \ell}(t)$ implies that

$$
r_{i}^{1, \ell}:=\left(\int_{-1}^{1}\left(Q_{i}^{1, \ell}(t)\right)^{2} d \mu_{n, \ell}(t)\right)^{-1}>0
$$

for $i=0,1, \ldots, k-1$. The three-term recurrence relation from Theorem 4.1 can be written as

$$
\left(t-a_{i}^{1, \ell}\right) Q_{i}^{1, \ell}(t)=b_{i}^{1, \ell} Q_{i+1}^{1, \ell}(t)+c_{i}^{1, \ell} Q_{i-1}^{1, \ell}(t),
$$

$i=1,2, \ldots, k-1$, where

$$
b_{i}^{1, \ell}=\frac{\eta_{i+1}^{1, \ell}}{\eta_{i}^{1, \ell}}>0, c_{i}^{1, \ell}=\frac{r_{i-1}^{1, \ell} b_{i-1}^{1, \ell}}{r_{i}^{1, \ell}}>0, a_{i}^{1, \ell}=1-b_{i}^{1, \ell}-c_{i}^{1, \ell} .
$$

The initial conditions are $Q_{0}^{1, \ell}(t)=1$ and

$$
Q_{1}^{1, \ell}(t)=\frac{n q(n q \ell+n q-2 n+2) t+A}{2 B},
$$

where

$$
\begin{aligned}
& A=n^{2}(q-1)(q \ell+q-2)+n(q \ell+5 q-6)-2(q-2), \\
& B=n^{2}(q-2)(q \ell+q-2)+2 n(q \ell+4 q-3)-4(q-2) .
\end{aligned}
$$

Finally in this description we note that by Theorem 4.1 the zeros of the polynomials $Q_{j}^{1, \ell}(t)$ interlace; i.e.,

$$
t_{j, i}^{1, \ell}<t_{j-1, i}^{1, \ell}<t_{j, i+1}^{1, \ell}, i=1,2, \ldots, j-1 .
$$

We conclude this section with a property of the polynomials $Q_{i}^{1, \ell}(t)$ which will give a particular answer to positive definiteness problems to arise below.

Lemma 4.3 If $\ell$ is as in (13), then $(t-\ell) Q_{i}^{1, \ell}(t) \in F_{>}$for every $i=0,1, \ldots, k-1$.

Proof. For every $i=0,1, \ldots, k-1$ it follows from (19) and (21) that

$$
(t-\ell) Q_{i}^{1, \ell}(t)=\frac{1-\ell}{1-q_{i}}\left(Q_{i+1}^{1,0}(t)-q_{i} Q_{i}^{1,0}(t)\right)
$$

where $q_{i}=Q_{i+1}^{1,0}(\ell) / Q_{i}^{1,0}(\ell)<0$ as in the proof of Theorem 4.2. Now $Q_{j}^{1,0}(t) \in F_{>}$(this is immediate from the definitions (6)-(17) $)$ completes the proof. 


\subsection{Construction and investigation of $Q_{k-1}^{1, \ell, s}(t)$ and Levenshtein-type polynomi- als}

In this section we perform the next step in our construction. Using the system $\left\{Q_{i}^{1, \ell}(t)\right\}_{i=0}^{k}$ from the previous section we derive explicitly polynomials $Q_{i}^{1, \ell, s}(t), i=1,2, \ldots, k-1$, orthogonal with respect to the measure $\mu_{n, \ell, s}(t)$. The last polynomial in this sequence, $Q_{k-1}^{1, \ell, s}(t)$, will be the main ingredient in our Levenshtein-type polynomials.

Consider the Christoffel-Darboux kernel associated with the polynomials $Q_{j}^{1, \ell}(t)$ :

$$
\begin{aligned}
R_{i}^{1, \ell}(x, y) & :=\sum_{j=0}^{i} r_{j}^{1, \ell} Q_{j}^{1, \ell}(x) Q_{j}^{1, \ell}(y) \\
& =r_{i}^{1, \ell} b_{i}^{1, \ell} \frac{Q_{i+1}^{1, \ell}(x) Q_{i}^{1, \ell}(y)-Q_{i+1}^{1, \ell}(y) Q_{i}^{1, \ell}(x)}{x-y}
\end{aligned}
$$

for $0 \leq i \leq k-1$ (when $x=y$ appropriate derivatives are used). Given (20) and assuming that

$$
t_{k, k}^{1,0}<s<t_{k, k}^{1, \ell}
$$

we define

$$
Q_{i}^{1, \ell, s}(t):=\frac{R_{i}^{1, \ell}(t, s)}{R_{i}^{1, \ell}(1, s)}, \quad i=0,1, \ldots, k-1 .
$$

We focus on the polynomials $Q_{k-1}^{1, \ell, s}(t)$. Their existence, uniqueness, and the correctness of the definition (25) follow as in Theorem 4.2. The proof of the next assertion about the zeros of $Q_{k-1}^{1, \ell, s}(t)$ is similar to the corresponding part of Theorem 4.2 but we include it for convenience of the reader. In addition to (18) we require

$$
\frac{Q_{k}^{1, \ell}(s)}{Q_{k-1}^{1, \ell}(s)}>\frac{Q_{k}^{1, \ell}(\ell)}{Q_{k-1}^{1, \ell}(\ell)}
$$

in order to get the smallest zero of $Q_{k-1}^{1, \ell, s}(t)$ in the interval $\left(\ell, t_{k, 1}^{1, \ell}\right)$.

Theorem 4.4 Let $n, q, \ell, s$, and $k$ be such that (20), (24), (18) and (26) are fulfilled. Then the polynomial $Q_{k-1}^{1, \ell, s}(t)$ has $k-1$ simple zeros $\alpha_{1}<\alpha_{2}<\cdots<\alpha_{k-1}$ such that

$$
\alpha_{1} \in\left(\ell, t_{k, 1}^{1, \ell}\right), \alpha_{i+1} \in\left(t_{k-1, i}^{1, \ell}, t_{k, i+1}^{1, \ell}\right),
$$

$i=1,2, \ldots, k-2$. In particular, $\ell<\alpha_{1}$ and $\alpha_{k-1}<s$.

Proof. It follows from the Christoffel-Darboux formula for $R_{i}^{1, \ell}$ and the definition (25) that the solutions of the equation

$$
\frac{Q_{k}^{1, \ell}(t)}{Q_{k-1}^{1, \ell}(t)}=\frac{Q_{k}^{1, \ell}(s)}{Q_{k-1}^{1, \ell}(s)}
$$


are the zeros of $Q_{k-1}^{1, \ell, s}(t)$ and the number $s$.

The rational function $Q_{k}^{1, \ell}(t) / Q_{k-1}^{1, \ell}(t)$ has $k-1$ simple poles at the zeros $t_{k-1, i}^{1, \ell}, i=1,2, \ldots, k-$ 1 , of $Q_{k-1}^{1, \ell}(t)$, and $k$ zeros at the zeros $t_{k, i}^{1, \ell}, i=1,2, \ldots, k$, of $Q_{k}^{1, \ell}(t)$. Therefore, there is a solution of (27); i.e., a zero of $Q_{k-1}^{1, \ell, s}(t)$, in each interval $\left(t_{k-1, i}^{1, \ell}, t_{k, i+1}^{1, \ell}\right), i=1,2, \ldots, k-2$, which accounts exactly for $k-2$ zeros, say $\alpha_{2}<\alpha_{3}<\cdots<\alpha_{k-1}$. Moreover, since $Q_{k}^{1, \ell}(s) / Q_{k-1}^{1, \ell}(s)<0$ under the assumptions for $s$, we actually have

$$
\alpha_{i+1} \in\left(t_{k-1, i}^{1, \ell}, t_{k, i+1}^{1, \ell}\right), i=2,3, \ldots, k-2 .
$$

Note that $\alpha_{k-1}<t_{k, k-1}^{1, \ell}<t_{k, k}^{1,0}<s$.

Since the function $Q_{k}^{1, \ell}(t) / Q_{k-1}^{1, \ell}(t)$ increases from $-\infty$ to $+\infty$ in the interval $\left[-\infty, t_{k-1,1}^{1, \ell}\right)$, the inequalities $0>Q_{k}^{1, \ell}(s) / Q_{k-1}^{1, \ell}(s)>Q_{k}^{1, \ell}(\ell) / Q_{k-1}^{1, \ell}(\ell)$ (see (26) $)$ imply that the smallest zero $\alpha_{1}$ of $Q_{k-1}^{1, \ell, s}(t)$ lies in the interval $\left(\ell, t_{k, 1}^{1, \ell}\right)$.

Finally, using again that $Q_{k}^{1, \ell}(s) / Q_{k-1}^{1, \ell}(s)<0$ and the fact that the function $Q_{k}^{1, \ell}(t) / Q_{k-1}^{1, \ell}(t)$ strictly increases from $-\infty$ to 1 for $t \in\left(t_{k-1, k-1}^{1, \ell}, 1\right]$, we have the root $s$ of (27) in this interval.

We can already define the Levenshtein-type polynomial

$$
f_{2 k}^{n, \ell, s}(t):=(t-\ell)(t-s)\left(Q_{k-1}^{1, \ell, s}(t)\right)^{2}
$$

and proceed with an investigation of its properties.

Denote by $L_{i}(t), i=0,1, \ldots, k+1$, the Lagrange basic polynomials generated by the nodes $\alpha_{0}<\alpha_{1}<\cdots<\alpha_{k-1}<\alpha_{k}<1$ and define

$$
\rho_{i}:=\int_{-1}^{1} L_{i}(t) d \mu_{n}(t), i=0,1, \ldots, k+1 .
$$

The next statement is an analog of one of the main theorems (Theorem 5.39) from [26]. It involves the zeros of $f_{2 k}^{n, \ell, s}(t)$ to form a right end-point Radau quadrature formula with positive weights.

Theorem 4.5 In the context of Theorem 4.4 let $\alpha_{0}:=\ell$ and $\alpha_{k}:=s$. Then the Radau quadrature formula

$$
f_{0}=\int_{-1}^{1} f(t) d \mu_{n}(t)=\rho_{k+1} f(1)+\sum_{i=0}^{k} \rho_{i} f\left(\alpha_{i}\right)
$$

is exact for all polynomials of degree at most $2 k$. Moreover, the weights $\rho_{i}, i=0, \ldots, k$, are positive, and $\rho_{k+1}>0$ provided $(t-\ell) Q_{k}^{1, \ell}(t) \in F_{>}$.

Proof. It follows from (29) that the formula (30) is exact for the Lagrange basis and hence for all polynomials of degree at most $k+1$. By a polynomial division, any polynomial $f(t)$ of degree at most $2 k$ can be written as

$$
f(t)=q(t)(t-\ell)(t-s)(1-t) Q_{k-1}^{1, \ell, s}(t)+g(t),
$$


where $\operatorname{deg}(q) \leq k-2$ and $\operatorname{deg}(g) \leq k+1$. Then the orthogonality of $Q_{k-1}^{1, \ell, s}(t)$ to all polynomials of degree at most $k-2$ with respect to the measure $d \mu_{n, \ell, s}(t)$ and the fact that the right-hand side of (30) is the same for $f(t)$ and $g(t)$ show the exactness of the quadrature formula (30) for $f(t)$.

We next employ the quadrature formula (30) to show the positivity of its weights $\rho_{i}$.

Using the polynomial $f(t)=(1-t)(t-\ell)\left(Q_{k-1}^{1, \ell, s}(t)\right)^{2}$ in (30) we obtain

$$
\rho_{k} f(s)=\int_{-1}^{1}\left(Q_{k-1}^{1, \ell, s}(t)\right)^{2} d \mu_{n, \ell}(t)>0
$$

because of the positive definiteness (up to degree $k-1$ ) of the measure $d \mu_{n, \ell}(t)$. Now $f(s)>0$ implies $\rho_{k}>0$. Similarly, with the polynomial $f(t)=(1-t)(s-t)\left(Q_{k-1}^{1, \ell, s}(t)\right)^{2}$ in (30) and the positive definiteness (up to degree $k-1$ ) of the measure $d \mu_{n, s}(t)$ (see Theorem 3.2) we conclude that $\rho_{0}>0$.

To see that $\rho_{i}>0$ for $i=1,2, \ldots, k-1$, we use the polynomials $f(t)=(1-t)(t-\ell)(s-$ t) $u_{k-1, i}^{2}(t)$, respectively, in (30), where $u_{k-1, i}(t)=Q_{k-1}^{1, \ell, s}(t) /\left(t-\alpha_{i}\right)$. Then $\operatorname{deg}\left(u_{k-1, i}\right)=k-2$ and the positive definiteness of $d \mu_{n, \ell, s}(t)$ (up to degree $k-2$ ) yields

$$
\rho_{i} f\left(\alpha_{i}\right)=\int_{-1}^{1} u_{k-1, i}^{2}(t) d \mu_{n, \ell, s}(t)>0 .
$$

Since $f\left(\alpha_{i}\right)>0$ for this choice of $f$, we derive that $\rho_{i}>0$.

Finally, we consider the weight $\rho_{k+1}$. In this case we use $f(t)=f_{2 k}^{n, \ell, s}(t)$ in (30) and find that

$$
f_{0}=\rho_{k+1} f(1)=\rho_{k+1}(1-s)(1-\ell) .
$$

Thus it is enough to see that the zeroth coefficient of $f_{2 k}^{n, \ell, s}(t)$ is positive. We use (25) to obtain that $f_{0}$ is equal to

$$
\begin{gathered}
\int_{-1}^{1}(t-\ell)(s-t)(1-t) Q_{k-1}^{1, \ell, s}(t) \frac{Q_{k-1}^{1, \ell, s}(t)-Q_{k-1}^{1, \ell, s}(1)}{t-1} d \mu_{n}(t) \\
+\int_{-1}^{1}(t-\ell)(t-s) Q_{k-1}^{1, \ell, s}(t) d \mu_{n}(t) \\
=\frac{1-s}{1-p_{k}} \int_{-1}^{1}(t-\ell)\left(Q_{k}^{1, \ell}(t)-p_{k} Q_{k-1}^{1, \ell}(t)\right) d \mu_{n}(t),
\end{gathered}
$$

where $p_{k}=Q_{k}^{1, \ell}(s) / Q_{k-1}^{1, \ell}(s)<0$. Then, under the assumption $(t-\ell) Q_{k}^{1, \ell}(t) \in F_{>}$and with $(t-\ell) Q_{k-1}^{1, \ell}(t) \in F_{>}$from Lemma 4.3 , it follows that the last integrand belongs to $F_{>}$and in particular its zeroth coefficient is positive. This completes the proof of the theorem.

Remark 4.6 The polynomials $f_{2 k}^{n, \ell, s}(t)$ can be also constructed via the system $\left\{Q_{i}^{1, s}(t)\right\}_{i=0}^{k}$ instead of $\left\{Q_{i}^{1, \ell}(t)\right\}_{i=0}^{k}$ in the sequence (11). Of course, the resulting system $\left\{Q_{i}^{n, \ell, s}(t)\right\}_{i=0}^{k-1}$ is the same.

\section{$5 \quad$ Bounding cardinalities and energies}

In the proof of the positive definiteness of his polynomials Levenshtein used (see [26, (3.88) and (3.92)]) what he called the strengthened Krein condition

$$
(t+1) Q_{i}^{1,1}(t) Q_{j}^{1,1}(t) \in F_{>}
$$


for every $i, j \in\{0,1, \ldots, n-3\}$. We need the following modification.

Definition 5.1 We say that the polynomials $\left\{Q_{i}^{1, \ell}(t)\right\}_{i=0}^{k}$ satisfy $(k, \ell)$-strengthened Krein condition if

$$
(t-\ell) Q_{i}^{1, \ell}(t) Q_{j}^{1, \ell}(t) \in F_{>}
$$

for every $i, j \in\{0,1, \ldots, k\}$ except possibly for $i=j=k$.

The strengthened Krein condition (32) holds true in $F_{q}^{n}$ for all admissible $i$ and $j$ (see [26, Lemma 3.25]). However, the $(k, \ell)$-strengthened Krein condition (33) is not true for every $\ell$, and for fixed $\ell$ is true only for relatively small $k$. On the other hand, for fixed $n$, all relevant pairs $(k, \ell)$ are finitely many and can be therefore subject to computational checks. Lemma 4.3 says that the condition is satisfied for all pairs $(i, 0), i=0,1, \ldots, k-1$.

The main result in this paper is the following. It includes our Levenshtein-type upper bound on $\mathcal{A}_{q}(n, \ell, s)$ as an analog of Theorem 5.42 of [26] and its counterpart, a universal lower bound on $\mathcal{E}_{h}(n, M, \ell)$ as an analog of the universal lower bound for $\mathcal{E}_{h}(n, M,-1)$ from [9]. We will use the notation

$$
S_{j}=\sum_{i=0}^{j} r_{i}=\sum_{i=0}^{j}(q-1)^{i}\left(\begin{array}{l}
n \\
i
\end{array}\right)
$$

for $j \in\{k-1, k, k+1\}$.

Theorem 5.2 Let $n, q, k, \ell$, and s satisfy the conditions (18), (20), (24), and (26) and suppose the $(k, \ell)$-strengthened Krein condition holds. Then the polynomial $f_{2 k}^{n, \ell, s}(t)$ belongs to $\mathcal{F}_{n, \ell, s}$ and, therefore,

$$
\mathcal{A}_{q}(n, \ell, s) \leq \frac{f_{2 k}^{n, \ell, s}(1)}{f_{0}}=\frac{1}{\rho_{k+1}}=L_{2 k}(n, \ell, s),
$$

where

$$
L_{2 k}(n, \ell, s):=\frac{S_{k}\left(Q_{k-1}^{1, \ell}(s)-Q_{k}^{1, \ell}(s)\right)}{\frac{r_{k+1} Q_{k+1}(\ell) Q_{k-1}^{1, \ell}(s)}{S_{k+1}\left(Q_{k+1}^{1,0}(\ell)-Q_{k}^{1,0}(\ell)\right)}-\frac{r_{k} Q_{k}(\ell) Q_{k}^{1, \ell}(s)}{S_{k-1}\left(Q_{k}^{1,0}(\ell)-Q_{k-1}^{1,0}(\ell)\right)}} .
$$

Furthermore, for fixed $\ell$, for $h$ being an absolutely monotone function on $[-1,1)$, and for $M$ determined by

the Hermite interpolan 1

$$
f_{2 k}^{n, \ell, s}(1)=M f_{0},
$$

$$
g_{2 k}^{n, \ell, M}(t):=H\left((t-s) f_{2 k}^{n, \ell, s}(t) ; h\right)
$$

belongs to $\mathcal{G}_{n, \ell}^{(h)}$, and, therefore,

$$
\begin{aligned}
\mathcal{E}_{h}(n, M, \ell) & \geq M\left(M g_{0}-g_{2 k}^{n, \ell, M}(1)\right) \\
& =M^{2} \sum_{i=0}^{k} \rho_{i} h\left(\alpha_{i}\right) .
\end{aligned}
$$

\footnotetext{
${ }^{1}$ The notation $g=H(f ; h)$ signifies that $g$ is the Hermite interpolant to the function $h$ at the zeros (taken with their multiplicity) of $f$.
} 
The bounds (34) and (35) can be attained only simultaneously by codes which have all their inner products in the roots of $f_{2 k}^{n, \ell, s}(t)$ and which are, in addition, $2 k$-design 2 in $F_{q}^{n}$.

Proof. It follows from the definitions (25) and (28) that the polynomial $f_{2 k}^{n, \ell, s}(t)$ can be written as

$$
c(t-\ell)\left(Q_{k}^{1, \ell}(t)+c_{1} Q_{k-1}^{1, \ell}(t)\right) \sum_{i=0}^{k-1} r_{i}^{1, \ell} Q_{i}^{1, \ell}(t) Q_{i}^{1, \ell}(s),
$$

where $r_{i}^{1, \ell}>0, i=0,1, \ldots, k-1$, and the constants $c=(1-s) /\left(1+c_{1}\right) R_{k-1}^{1, \ell}(1, s)$ and $c_{1}=$ $-Q_{k}^{1, \ell}(s) / Q_{k-1}^{1, \ell}(s)$ are positive under the assumptions for $\ell$ and $s$. Since $Q_{i}^{1, \ell}(s)>0$ for $0 \leq$ $i \leq k-1$, the polynomial $f_{2 k}^{n, \ell, s}(t)$ becomes a positive linear combination of polynomials $(t-$ $\ell) Q_{i}^{1, \ell}(t) Q_{j}^{1, \ell}(t)$, where $i \in\{k, k-1\}$ and $j \leq k-1$. Therefore $f_{2 k}^{n, \ell, s}(t) \in F_{>}$because of the $(k, \ell)$-strengthened Krein condition. This and the obvious $f_{2 k}^{n, \ell, s}(t) \leq 0$ for every $t \in[\ell, s]$ implies that $f_{2 k}^{n, \ell, s}(t) \in F_{n, \ell, s}$.

To compute the ratio $f_{2 k}^{n, \ell, s}(1) / f_{0}$ we write $f_{0}$ as in (31) and then use the representation of $(t-\ell) Q_{j}^{1, \ell}(t)$ by the Christoffel-Darboux formula (see (19) and (21)) for $j=k-1$ and $k$. The integrand becomes a linear combination of the polynomials $Q_{i}^{1,0}(t), i=k-1, k, k+1$. Since

$$
\int_{-1}^{1} Q_{j}^{1,0}(t) d \mu_{n}(t)=\int_{-1}^{1} \frac{T_{j}(t, 1)}{T_{j}(1,1)} d \mu_{n}(t)=\frac{1}{S_{j}}
$$

after simplifications we obtain the explicit form of the bound (34).

We proceed with the energy bound. Denote by $t_{1} \leq t_{2} \leq \cdots \leq t_{2 k}$ the zeros of $f_{2 k}^{n, \ell, s}(t)$ in increasing order and counting their multiplicity; i.e., $t_{1}:=\alpha_{0}=\ell, t_{2 i}=t_{2 i+1}:=\alpha_{i}, i=$ $1, \ldots, k-1$, and $t_{2 k}:=\alpha_{k}=s$. Then the Newton interpolation formula gives that the polynomial $g_{2 k}^{n, \ell, M}(t)$ is a linear combination with nonnegative coefficients of the constant 1 and the partial products

$$
\prod_{j=1}^{m}\left(t-t_{j}\right), \quad m=1,2, \ldots, 2 k .
$$

Since $t_{2 i}, i=1, \ldots, k$, are the roots of $Q_{k}^{1, \ell}(t)+\alpha Q_{k-1}^{1, \ell}(t)$ (see (25)) it follows from [14, Theorem 3.1] that the partial products $\prod_{j=1}^{m}\left(t-t_{2 j}\right), m=1, \ldots, k-1$, have positive coefficients when expanded in terms of the polynomials $Q_{i}^{1, \ell}(t), i=0,1, \ldots, k-1$. Therefore $g_{2 k}^{n, \ell, M}(t)$ is a linear combination with positive coefficients of terms $(t-\ell) Q_{i}^{1, \ell}(t) Q_{j}^{1, \ell}(t), i, j \in\{0,1, \ldots, k-1\}$, and the last partial product which is in fact $f_{2 k}^{n, \ell, s}(t)$. Now $g_{2 k}^{n, \ell, M}(t) \in F_{>}$follows from the validity of the $(k, \ell)$-strengthened Krein condition and from $f_{2 k}^{n, \ell, s}(t) \in F_{>}$, obtained in the first part of the proof.

Multiple application of the Rolle's theorem implies that $g_{2 k}^{n, \ell, M}(t) \leq h(t)$ for every $t \in[\ell, 1)$ and therefore $g_{2 k}^{n, \ell, M}(t) \in \mathcal{G}_{n, \ell}^{(h)}$. The explicit form of the bound (35) via the weights $\rho_{i}$ and the nodes

\footnotetext{
${ }^{2}$ Also known as orthogonal arrays of strength $2 k$.
} 
$\alpha_{i}$ follows from the quadrature formula (30) applied for $g_{2 k}^{n, \ell, M}(t)$ and the interpolation conditions $g_{2 k}^{n, \ell, M}\left(\alpha_{i}\right)=h\left(\alpha_{i}\right), i=0,1, \ldots, k$.

There are two kinds of conditions for attaining the general linear programming bounds (2) and (3) (see, for example, [25, Eqs. (32)-(33)] for (2)). First, the inner products of distinct points of any attaining code must be among the zeros of the polynomial $f(t)$ in (2) or the abscissas of the touching/intersection points of the polynomial $g(t)$ and the potential function $h(t)$ in (3). Second, the complementary slackness conditions $f_{i} B_{i}^{\prime}=0$ (or $g_{i} B_{i}^{\prime}=0$ ) for $i=1,2, \ldots, n$, where $\left(B_{0}^{\prime}, B_{1}^{\prime}, \ldots, B_{n}^{\prime}\right)$ is the MacWilliams transform of the attaining code, have to be satisfied.

By our construction, the roots of the polynomial $f_{2 k}^{n, \ell, s}(t)$ coincide exactly with the abscissas of the touching/intersection points of the graphs of $g_{2 k}^{n, \ell, M}(t)$ and $h(t)$. Further, $f_{2 k}^{n, \ell, s}(t) \in F_{>}$ implies (and $g_{2 k}^{n, \ell, M}(t) \in F_{>}$does as well) that $B_{i}^{\prime}=0$ for $i=1,2, \ldots, 2 k$; i.e., any attaining code has to be a $2 k$-design.

Therefore, the bounds (34) and (35) can be attained only simultaneously by codes which have all their inner products in the roots of $f_{2 k}^{n, \ell, s}(t)$ (equivalently, in the abscissas of the touching/intersection points of the graphs of $g_{2 k}^{n, \ell, M}(t)$ and $\left.h(t)\right)$ and which are, in addition, $2 k$-designs in $F_{q}^{n}$. This completes the proof.

The bound (34) was obtained and investigated for $k=1$ (in our notations) and the corresponding $\ell$ and $s$ by Helleseth, Kløve and Levenshtein [21. In that paper, comparisons with the Levenshtein bound (see [25]) obtained by polynomials of degrees 2 and 3, and detailed descriptions of all known codes attaining $L_{2}(n, \ell, s)$ can be found. We discuss some examples from [21] in Section 10. The bound (35) for $k=1$ is given by

$$
E_{h}(n, M, \ell) \geq M^{2}\left(\rho_{0} h(\ell)+\rho_{1} h(s),\right.
$$

where $\rho_{0}$ and $\rho_{1}$ can be computed as shown in Example 6.3 below.

For $k>1$, it does not seem customary to consider the bounds (34) and (35) for fixed $k$ and varying $\ell$ and $s$. Instead, in Sections 7 and 10.1 we describe them as a system of bounds for fixed $\ell>-1$ and varying $k=1,2, \ldots$ and corresponding $s \in I_{k}^{(\ell)} \subset\left(t_{k-1, k-1}^{1, \ell}, 1\right)$, like the Levenshtein bound is described with fixed $\ell=-1$.

The optimality of the bounds (34) and (35) will be discussed in Section 7

Remark 5.3 The above proof of the bound (35) does not require $M$ to be integer. In particular, the expression at the right hand side of (35) is defined for any real $M \in\left[2, q^{n}\right]$. This is customary in certain investigations.

\section{Codes attaining the bounds - conditions, distance distributions}

Like in the case $\ell=-1$ (see Theorem 5.55 and Remark 5.58 in [26]; also [6] for details), codes which attain the bounds from Theorem 5.2 have special combinatorial and geometric properties. Also, it is important that the bounds (34) and (35) can be attained only simultaneously since the conditions of their attaining coincide.

The conditions $\alpha_{i} \in T_{n}, i=1,2, \ldots, k-1$, are quite restrictive. For example, they say that all roots of $Q_{k-1}^{1, \ell, s}(t)$ belong to $T_{n}$. In particular, the roots of $Q_{k-1}^{1, \ell, s}(t)$ must be all rational which 
is usually a good starting point for deep algebraic investigation. However, in this section we focus on the combinatorial meaning of the fact that all inner products of attaining codes must belong to the set $\left\{\alpha_{0}, \alpha_{1}, \ldots, \alpha_{k}\right\}$.

Definition 6.1 Let $C \subset F_{q}^{n}$ be a code. For fixed $x \in C$ and $t_{n-i} \in T_{n}, i \in\{0,1, \ldots, n\}$, denote by

$$
A_{i}(x):=\left|\left\{y \in C:\langle x, y\rangle=t_{n-i}\right\}\right|,
$$

the number of the points of $C$ at distance $i$ from $x$. The system of nonnegative integers $\left(A_{i}(x)\right.$ : $i=0,1, \ldots, n)$ is called distance distribution of $C$ with respect to $x$.

It is clear that $A_{0}(x)=1$ and that $A_{i}(x) \neq 0$ is possible only for $i \in\{d, d-1, \ldots, D-1, D\}$, where $d$ and $D$ are the minimum and maximum distance of $C$, respectively (recall that $s=1-2 d / n$ and $\ell=1-2 D / n)$. We show that for codes attaining (34) and (35) the whole distance distribution can be computed.

When dealing with distance distributions, it is convenient to use the following characteristic property of designs in polynomial metric spaces (see [25] for Hamming spaces; Equation (1.10) in [20] for the general case of polynomial metric spaces). A code $C \subset F_{q}^{n}$ is a $\tau$-design if and only if

$$
\sum_{y \in C} f(\langle x, y\rangle)=f_{0}|C|
$$

holds for every $x \in F_{q}^{n}$ and every real polynomial $f(t)$ of degree at most $\tau$.

Theorem 6.2 If a code $C \subset C_{n, q}(\ell, s)$ attains the bounds (34) and (35), then its distance distribution with respect to any point $x \in C$ does not depend on the choice of $x$ and can be computed from a system of linear equations. Explicitly, we have

$$
A_{\alpha_{i}}=A_{\alpha_{i}}(x)=\rho_{i}|C|\left(=\frac{\rho_{i}}{\rho_{k+1}}\right), i=0,1, \ldots, k .
$$

Proof. Let $C$ be a code that attains the bounds (34) and (35). By Theorem 5.2 the code $C$ is a $2 k$-design, so (36) holds. Fixing $x \in C$ and grouping the terms with the same $t_{n-i}$ in the left hand side we write (36) as

$$
1+\sum_{i=d}^{D} A_{i}(x) f\left(t_{n-i}\right)=f_{0}|C| .
$$

In our case $A_{i}(x) \neq 0$ is possible only if $t_{n-i} \in\left\{\alpha_{0}=\ell, \alpha_{1}, \ldots, \alpha_{k-1}, s=\alpha_{k}\right\}$ (see Theorem 5.2). Setting consecutively $f(t)=1, t, t^{2}, \ldots, t^{k}$ in (37) yields the Vandermonde-type system

$$
1+\sum_{i=0}^{k} A_{\alpha_{i}}(x) \alpha_{i}^{u}=b_{u}|C|, u=0,1, \ldots, k,
$$

where

$$
b_{u}=\int_{-1}^{1} t^{u} d \mu_{n}
$$


is the zeroth coefficient in the Krawtchouk expansion of $t^{u}, u=0,1, \ldots, k$.

Since the solution of (38) is unique, it follows that the distance distribution $\left\{A_{\alpha_{i}}(x): i=\right.$ $0,1, \ldots, k\}$ does not depend on the choice of $x \in C$ and can be computed from the system (38) (so it is uniquely determined by the parameters $n, q, \ell, s$, and $|C|=L_{2 k}(n, \ell, s)$ ). Thus we can omit $x$ in the notation of the distance distributions of $C$.

The combination of (38) and the quadrature formula (30) gives explicit formulas for the distance distributions. Indeed, setting (again!) the polynomials $f(t)=1, t, t^{2}, \ldots, t^{k}$ in (30) produces the system

$$
\rho_{k+1}+\sum_{i=0}^{k} \rho_{i} \alpha_{i}^{u}=b_{u}, u=0,1, \ldots, k .
$$

Multiplying all equations of (39) by $|C|$ and taking into account that

$$
\rho_{k+1}|C|=\rho_{k+1} L_{2 k}(n, \ell, s)=1
$$

by (34), we obtain the system (38) again but with unknowns $\rho_{i}|C|$. The solutions of both systems must coincide; i.e., $A_{\alpha_{i}}=\rho_{i}|C|, i=0,1, \ldots, k$, as required.

Of course, the formulas from Theorem 6.2 have to produce nonnegative integers. Thus they in fact yield strong necessary conditions for existence of codes attaining (34) and (35).

The above approach works (to some extent) also for the external (when $x \in F_{q}^{n} \backslash C$ ) distance distributions of $C$ with respect to $x$. Then it yields a system of $2 k+1$ equations with respect to $n$ unknowns $A_{1}(x), A_{2}(x), \ldots, A_{n}(x)$ (note that $A_{0}(x)=0$ ). Typically, $n$ is quite larger than $2 k+1$ and our system has many solutions. However, the solutions belong to a finite set and it is possible to find them for subsequent analysis (see, for example, [11,12]). Such computations could yield upper bounds on the covering radius of codes attaining (34) and (35). In fact, this approach works in general for designs in $F_{q}^{n}$ as well (see [11,12]).

We remark also that the computations of distance distributions of attaining codes allows easy derivation of the energy of these codes.

Example 6.3 We show how Theorem 6.2 works for $k=1$. Assume that $C \subset C_{n, q}(\ell, s)$ attains the bound $L_{2}(n, \ell, s)$. Then the system (38) (for $k=1$ ) is solved explicitly as follows. We have $b_{1}=(2-q) / q$ and (38) becomes

$$
\mid \begin{aligned}
& A_{\ell}+A_{s}=|C|-1 \\
& \ell A_{\ell}+s A_{s}=\frac{(2-q)|C|}{q}-1,
\end{aligned}
$$

whence we obtain

$$
\begin{aligned}
& A_{\ell}=\frac{q(1+s)(|C|-1)-2|C|}{q(s-\ell)}, \\
& A_{s}=\frac{2|C|-q(1+\ell)(|C|-1)}{q(s-\ell)} .
\end{aligned}
$$

Now $\rho_{0}=A_{\ell} /|C|$ and $\rho_{s}=A_{s} /|C|$ are computed in turn and the energy of $C$ (attaining the bound (35)) is given by

$$
\begin{aligned}
E_{h}(C) & =|C|^{2}\left(\rho_{0} h(\ell)+\rho_{1} h(s)\right) \\
& =|C|\left(A_{\ell} h(\ell)+A_{s} h(s)\right) .
\end{aligned}
$$




\section{On the optimality of the bounds (34) and (35)}

In this section we assume that $n, q, k$, and $\ell \in\left(t_{k+1,1}^{1,0}, t_{k, 1}^{1,0}\right)$ are fixed and there exists an interval $I_{k}^{(\ell)} \subset\left(t_{k, k}^{1,0}, 1\right)$ such that for every $s \in I_{k}^{(\ell)}$ the bounds (34) and (35) are optimal in the following sense

$$
L_{2 k}(n, \ell, s)=\min \left\{L_{2 j}(n, \ell, s): j \geq 1, s \in I_{k}^{(\ell)}\right\} ;
$$

i.e., the bound $L_{2 k}(n, \ell, s)$ is optimal for every $s \in I_{k}^{(\ell)}$ among all bounds $L_{2 j}(n, \ell, s)$ (if any with $j \neq k$ ). Then the image of $I_{k}^{(\ell)}$ under the function $L_{2 k}(n, \ell, s)$ (which is continuous in $s$ ) is a subinterval of $\left(L_{2 k}\left(n, \ell, t_{k, k}^{1,0}\right), q^{n}\right)$ denoted by $J_{2 k}^{(\ell)}$.

For $s \in I_{k}^{(\ell)}$ and positive integer $j$, we define

$$
R_{j}^{n, \ell}(s):=\frac{1}{L_{2 k}(n, \ell, s)}+\sum_{i=0}^{k} \rho_{i} Q_{j}\left(\alpha_{i}\right) .
$$

Similarly, for $M \in J_{2 k}^{(\ell)}$ and positive integer $j$, we define

$$
S_{j}^{n, \ell}(M):=\frac{1}{M}+\sum_{i=0}^{k} \rho_{i} Q_{j}\left(\alpha_{i}\right),
$$

where the parameters $\left(\rho_{i}, \alpha_{i}\right)_{i=0}^{k}$ come from fixing $s \in I_{k}^{(\ell)}$ by the equality $M=L_{2 k}(n, \ell, s)$. Note that the values of $S_{j}^{n, \ell}(M)$ for integers $M \in J_{2 k}^{(\ell)}$ are just particular values of $R_{j}^{n, \ell}(s)$ for $s \in I_{k}^{(\ell)}$. On the other hand, it is clear that $M$ could be considered as real variable whenever this facilitates an analysis.

Remark 7.1 The quadrature formula (30) applied for $Q_{j}^{(n, q)}(t)$ with $1 \leq j \leq 2 k$ implies immediately that $R_{j}^{n, \ell}(s)=0$ for all $s \in I_{k}^{(\ell)}$ and that $S_{j}^{n, \ell}(M)=0$ for all $M \in J_{2 k}^{(\ell)}$. Thus we sometimes assume (to avoid trivialities) in what follows that $j \geq 2 k+1$.

The next theorem gives necessary and sufficient conditions for existence of better bounds than (34) and (35) (obtained by polynomials from $\mathcal{F}_{n, \ell, s}$ and $\mathcal{G}_{n, \ell}^{(h)}$, respectively). Its part (a) is a counterpart of Theorem 5.47 from [26] (see also [7, Theorem 3.1]) and its part (b) is a counterpart of Theorem 5.1 from 9 .

Theorem 7.2 (a) Given $n, q, \ell, k$, and $s \in I_{k}^{(\ell)}$, the bound (34) can be improved by a polynomial from $\mathcal{F}_{n, \ell, s}$ if and only if there exists a positive integer $j \geq 2 k+1$ such that $R_{j}^{n, \ell}(s)<0$. In particular, if $R_{j}^{n, \ell}(s) \geq 0$ for every $j \leq m$ then (34) cannot be improved by a polynomial from $\mathcal{F}_{n, \ell, s}$ of degree at most $m$.

(b) Given $n, q, \ell, k, M \in J_{2 k}^{(\ell)}$, and a strictly absolutely monotone $h$, the bound (35) can be improved by a polynomial from $\mathcal{G}_{n, \ell}^{(h)}$ if and only if there exists a positive integer $j \geq 2 k+1$ such that $S_{j}^{n, \ell}(M)<0$. In particular, if $S_{j}^{n, \ell}(M) \geq 0$ for every $j \leq m$ then (35) cannot be improved by a polynomial from $\mathcal{G}_{n, \ell}^{(h)}$ of degree at most $m$. 
Proof. (a) Assume that $R_{j}^{n, \ell}(s) \geq 0$ for every positive integer $j$. Let $f(t) \in \mathcal{F}_{n, \ell, s}$ and

$$
f(t)=u(t)+\sum_{j \geq 2 k+1} f_{j} Q_{j}(t),
$$

where $u(t)$ has degree at most $2 k$ and zeroth coefficient $u_{0}$. Note that $f\left(\alpha_{i}\right) \leq 0$ for $i=0,1, \ldots, k$, $f_{j} \geq 0$ for $j \geq 2 k+1$, and $f_{0}=u_{0}$. Applying (30) to $u(t)$ and using that $L_{2 k}(n, \ell, s)=1 / \rho_{k+1}$, we obtain

$$
\begin{aligned}
f_{0} & =u_{0}=\frac{u(1)}{L_{2 k}(n, \ell, s)}+\sum_{i=0}^{k} \rho_{i} u\left(\alpha_{i}\right) \\
& =\frac{f(1)}{L_{2 k}(n, \ell, s)}+\sum_{i=0}^{k} \rho_{i} f\left(\alpha_{i}\right)-\sum_{j \geq 2 k+1} f_{j} R_{j}^{n, \ell}(s) \\
& \leq \frac{f(1)}{L_{2 k}(n, \ell, s)}
\end{aligned}
$$

Therefore, $f(1) / f_{0} \geq L_{2 k}(n, \ell, s)$; i.e., $f(t)$ does not produce better bound than (34).

Let, conversely, $R_{j}^{n, \ell}(s)<0$ for some $j \geq 2 k+1$. We construct a degree $j$ improving polynomial of the form

$$
v(t)=(a(t)+c) f_{2 k}^{n, \ell, s}(t)=\sum_{i=0}^{j} v_{i} Q_{i}(t),
$$

where the number $c$ and the polynomial $a(t)$ of degree $j-2 k$ will be properly chosen.

The polynomial $a(t)$ is immediate - applying polynomial division we consider the unique polynomials $a(t)$ (quotient) and $b(t)$ (remainder) such that

$$
Q_{j}(t)=a(t) f_{2 k}^{n, \ell, s}(t)+b(t)
$$

where the remainder $b(t)$ has degree at most $2 k-1$. Let

$$
b(t)=\sum_{i=0}^{2 k-1} b_{i} Q_{i}(t), \quad f_{2 k}^{n, \ell, s}(t)=\sum_{i=0}^{2 k} f_{i} Q_{i}(t)
$$

be the Krawtchouk expansions of $b(t)$ and $f_{2 k}^{n, \ell, s}(t)$, respectively. Then it is easy to see that

$$
c:=\max \left\{-\min _{t \in[\ell, s]} a(t), \max _{0 \leq i \leq 2 k-1} \frac{b_{i}}{f_{i}}, 0\right\}
$$

(recall that $f_{i}>0$ for every $i=0,1, \ldots, 2 k$ ) guaranties that $v(t) \in \mathcal{F}_{n, \ell, s}$.

Since the polynomial

$$
v(t)-Q_{j}(t)=c f_{2 k}^{n, \ell, s}(t)-b(t)
$$


has degree at most $2 k$, its zeroth coefficient $v_{0}$ (which coincides with the zeroth coefficient of $v(t)$ ) can be computed from (30). We have consecutively

$$
\begin{aligned}
v_{0} & =\frac{c f_{2 k}^{n, \ell, s}(1)-b(1)}{L_{2 k}(n, \ell, s)}+\sum_{i=0}^{k} \rho_{i}\left(c f_{2 k}^{n, \ell, s}\left(\alpha_{i}\right)-b\left(\alpha_{i}\right)\right) \\
& =\frac{v(1)-1}{L_{2 k}(n, \ell, s)}-\sum_{i=0}^{k} \rho_{i} Q_{j}\left(\alpha_{i}\right) \\
& =\frac{v(1)}{L_{2 k}(n, \ell, s)}-R_{j}^{n, \ell}(s)>\frac{v(1)}{L_{2 k}(n, \ell, s)}
\end{aligned}
$$

Therefore $v(1) / v_{0}<L_{2 k}(n, \ell, s)$; i.e., $v(t)$ improves on $L_{2 k}(n, \ell, s)$, which completes the proof of the sufficiency.

(b) Suppose that $S_{j}^{n, \ell}(M) \geq 0$ for every positive integer $j$. Any polynomial $g(t) \in \mathcal{G}_{n, \ell}^{(h)}$ can be written as

$$
g(t)=u(t)+\sum_{j \geq 2 k+1} g_{j} Q_{j}(t)
$$

for some polynomial $u(t)$ of degree at most $2 k$ with zeroth coefficient $u_{0}$. We have $g\left(\alpha_{i}\right) \leq h\left(\alpha_{i}\right)$ for $i=0,1, \ldots, k, g_{j} \geq 0$ for every $j \geq 2 k+1$, and $g_{0}=u_{0}$. Therefore, using (30) for $u(t)$ (recall that $\left.M=L_{2 k}(n, \ell, s)\right)$, we consecutively obtain

$$
\begin{aligned}
& M g_{0}-g(1)=M u_{0}-g(1) \\
= & M \sum_{i=0}^{k} \rho_{i} u\left(\alpha_{i}\right)-\sum_{j \geq 2 k+1} g_{j} \\
= & M \sum_{i=0}^{k} \rho_{i}\left(g\left(\alpha_{i}\right)-\sum_{j \geq 2 k+1} g_{j} Q_{j}\left(\alpha_{i}\right)\right)-\sum_{j \geq 2 k+1} g_{j} \\
= & M \sum_{i=0}^{k} \rho_{i} g\left(\alpha_{i}\right)-M \sum_{j \geq 2 k+1} g_{j}\left(\frac{1}{M}+\sum_{i=0}^{k} \rho_{i} Q_{j}\left(\alpha_{i}\right)\right) \\
= & M \sum_{i=0}^{k} \rho_{i} g\left(\alpha_{i}\right)-M \sum_{j \geq 2 k+1} g_{j} S_{j}^{n, \ell}(M) \\
\leq & M \sum_{i=0}^{k} \rho_{i} h\left(\alpha_{i}\right),
\end{aligned}
$$

where, for the last inequality, we used $S_{j}^{n, \ell}(M) \geq 0$ for $j \geq 2 k+1$. Hence the bound, produced by $g(t)$, does not improve on (35).

Conversely, assume that $h$ is strictly absolutely monotone and suppose that $S_{j}^{n, \ell}(M)<0$ for some positive integer $j \geq 2 k+1$. We are going to improve (35) by using a polynomial

$$
v(t)=\varepsilon Q_{j}(t)+a(t)=\sum_{i=0}^{j} v_{i} Q_{i}(t),
$$


where the number $\varepsilon>0$ and the polynomial $a(t)$ of degree at most $2 k$ will be properly chosen.

Denote

$$
\tilde{h}(t):=h(t)-\varepsilon Q_{j}(t)
$$

and select $\varepsilon$ such that $\tilde{h}^{(i)}(t) \geq 0$ on $[\ell, 1]$ for all $i=0,1, \ldots, j$. This choice of $\varepsilon$ is possible since the function $h$ is strictly absolutely monotone. Since $\tilde{h}^{(i)}(t)=h^{(i)}(t)>0$ for $i>j$ the function $\tilde{h}(t)$ is absolutely monotone.

Now the polynomial $a(t)$ is chosen to be the Hermite interpolant of the new function $\tilde{h}$ at the nodes $\ell=\alpha_{0}$ (simply) and $\alpha_{i}, i=1,2, \ldots, k$, (doubly) exactly as the original $g_{2 k}^{n, \ell, M}(t)$ does. Then we can infer as in Theorem 5.2 that $a(t) \in \mathcal{G}_{n, \ell}^{(\tilde{h})}$ implying that $v(t) \in \mathcal{G}_{n, \ell}^{(h)}$.

It remains to prove that $v(t)$ gives a bound which is better than (35) indeed. Let

$$
a(t)=\sum_{i=0}^{2 k-1} a_{i} Q_{i}(t)
$$

and note that $v_{0}=a_{0}$ and $v(1)=a(1)+\varepsilon$. We multiply by $\rho_{i}$ and sum up the interpolation equalities for $a(t)$ to compute

$$
\sum_{i=0}^{k} \rho_{i} a\left(\alpha_{i}\right)=\sum_{i=0}^{k} \rho_{i} h\left(\alpha_{i}\right)-\varepsilon \sum_{i=0}^{k} \rho_{i} Q_{j}\left(\alpha_{i}\right) .
$$

Since

$$
M \sum_{i=0}^{k} \rho_{i} a\left(\alpha_{i}\right)=M a_{0}-a(1)
$$

by (30) and

$$
M \sum_{i=0}^{k} \rho_{i} Q_{j}\left(\alpha_{i}\right)=M S_{j}^{n, \ell}(M)-1
$$

by the definition of the function $S_{j}^{n, \ell}(M)$, we obtain

$$
M a_{0}-a(1)=M \sum_{i=0}^{k} \rho_{i} h\left(\alpha_{i}\right)+\varepsilon-\varepsilon M S_{j}^{n, \ell}(M)
$$

which yields

$$
\begin{aligned}
M v_{0}-v(1) & =M \sum_{i=0}^{k} \rho_{i} h\left(\alpha_{i}\right)-\varepsilon M S_{j}^{n, \ell}(M) \\
& >M \sum_{i=0}^{k} \rho_{i} h\left(\alpha_{i}\right) .
\end{aligned}
$$

The last inequality means that the polynomial $v(t)$ gives better than (35) bound.

Remark 7.1 and Theorem 7.2 give the following optimality property of the bounds (34) and (35). 
Corollary 7.3 None of the bounds (34) and (35) can be improved by using polynomials from $\mathcal{F}_{n, \ell, s}$ and $\mathcal{G}_{n, \ell}^{(h)}$, respectively, of degree at most $2 k$.

The corresponding optimality results for the case $\ell=-1$ were proved for the maximum code problem by Sidelnikov [27] (see also [24]) and for the minimum energy problem by the authors [9].

We provide another formula for the test functions. We use the notations

$$
Q_{j}(t):=\sum_{i=0}^{j} a_{j, i} t^{i}
$$

for the coefficients of the Krawtchouk polynomials,

$$
S_{u}=\frac{1}{L_{2 k}(n, \ell, s)}+\sum_{i=0}^{k} \rho_{i} \alpha_{i}^{u},
$$

and recall that $b_{u}:=\int_{-1}^{1} t^{u} d \mu_{n}$ as in the proof of Theorem 6.2

Lemma 7.4 With the above notations,

$$
\sum_{i=0}^{j} a_{j, i} b_{i}=0
$$

for very positive integer $j$.

Proof. This is the zeroth coefficient of $Q_{j}(t)$ which is, of course, equal to 0 .

Lemma 7.5 With the above notations,

$$
b_{u}=S_{u}
$$

for every $u=0,1, \ldots, 2 k$.

Proof. This follows from the quadrature formula (30) applied with the polynomial $t^{u}$.

Theorem 7.6 For every $s \in I_{k}^{(\ell)}$ and positive integer $j>2 k$,

$$
R_{j}^{n, \ell}(s)=\sum_{u=2 k+1}^{j} a_{j, u}\left(S_{u}-b_{u}\right)
$$

and, correspondingly,

$$
S_{j}^{n, \ell}(M)=\sum_{u=2 k+1}^{j} a_{j, u}\left(S_{u}-b_{u}\right)
$$

with parameters coming from $M=L_{2 k}(n, \ell, s) \in J_{2 k}^{(\ell)}$ as in Theorem 5.2. 
Proof. It is enough to prove the formula for $R_{j}^{n, \ell}(s)$. Grouping the powers of $\alpha_{i}$ in the definition of $R_{j}^{n, \ell}(s)$ and using Lemma 7.4 yield

$$
R_{j}^{n, \ell}(s)=\sum_{u=0}^{j} a_{j, u}\left(S_{u}-b_{u}\right)
$$

Now Lemma 7.5 implies the required identity.

For fixed $n, q, \ell$ and $k$, there are only finitely many $s \in T_{n} \cap I_{k}^{(\ell}$ and finitely many $M \in J_{k}^{(\ell)}$. Thus a numerical investigation of the signs of the functions $R_{j}^{n, \ell}(s)$ and $S_{j}^{n, \ell}(M)$ can be performed.

We conclude this section with a few comments on the possibility for using higher degree polynomials.

Corollary 7.3 implies (like in the case $\ell=-1$ ) that improvements of the bounds (34) and (35) by polynomials are only possible for degrees higher than $2 k$. We refer to (34) and (35) to as first level bounds and call second level bounds any improvement by polynomials from $\mathcal{F}_{n, \ell, s}$ or $\mathcal{G}_{n, \ell}^{(h)}$.

In the proof of Theorem 7.2 we, in fact, produced improving polynomials. However, the numerical experiments show that these are marginal and are never optimal like the first levels are. A detailed second level universal bounds based on Levenshtein-type quadratures that generalize (30) will be developed in a future work (see [10] for the spherical codes case when $\ell=-1$ ).

\section{On optimal linear programming results}

Corollary 7.3 shows that the bounds (34) and (35) cannot be improved by using polynomials from $\mathcal{F}_{n, \ell, s}$ and $\mathcal{G}_{n, \ell}^{(h)}$, respectively, of degree at most $2 k$. However, the requirements $f(t) \leq 0$ (or $g(t) \leq$ $h(t)$, respectively) for every $t \in[\ell, s]$ (for every $t \in[\ell, 1]$, respectively) are stronger than really necessary. What we need in fact, is $f(t) \leq 0$ (or $g(t) \leq h(t)$, respectively) for every $t \in[\ell, s] \cap T_{n}$ (for every $t \in[\ell, 1) \cap T_{n}$, respectively). Of course, we always have $\{\ell, s\}=\left\{t_{n-D}, t_{n-d}\right\} \subset T_{n}$, but the roots $\alpha_{1}, \alpha_{2}, \ldots, \alpha_{k-1}$ of the polynomial $f_{2 k}^{n, \ell, s}(t)$ are not necessarily in the set $T_{n}$. This makes a difference for $k>1$ allowing a natural relaxation of our linear programming problems.

We describe a modification of the polynomials $f_{2 k}^{n, \ell, s}(t)$ and $g_{2 k}^{n, M, \ell}(t)$ which is going to produce better bounds provided the new polynomials are still good for linear programming.

We replace the double roots $\alpha_{1}, \alpha_{2}, \ldots, \alpha_{k-1}$ (the touching points, respectively) with their closest neighbours from $T_{n}$. More precisely, if $\alpha_{i} \in\left(t_{j-1}, t_{j}\right)$ for some integer $j \in\{n-D, n-D+$ $1, \ldots, n-d\}$, then we replace the double zero $\alpha_{i}$ of $f_{2 k}^{n, \ell, s}(t)$ by two simple zeros $\gamma_{2 i-1}=t_{j-1}$ and $\gamma_{2 i}=t_{j}$. If $\alpha_{i}=t_{j}$, then one can try both $\left(\gamma_{2 i-1}, \gamma_{2 i}\right)=\left(t_{j-1}, t_{j}\right)$ and $\left(t_{j}, t_{j+1}\right)$. Finally, setting $\gamma_{0}:=\ell$ and $\gamma_{2 k-1}:=s$, we define our refining polynomial for the maximum code problem to be

$$
f_{\mathrm{ref}}(t):=\prod_{i=0}^{2 k-1}\left(t-\gamma_{i}\right)=\sum_{i=0}^{2 k} f_{i} Q_{i}(t)
$$

Then

$$
g_{\mathrm{ref}}(t):=H\left(f_{\mathrm{ref}}(t)\left(t-t_{n-d-1}\right) ; h\right)=\sum_{i=0}^{2 k} g_{i} Q_{i}(t)
$$


will be our refining polynomial for the minimum energy problem. Note that in $g_{\text {ref }}$ we may have intersections at $t_{n-d}$ and at $t_{n-d-1}$ or $t_{n-d+1}$ instead of touching at $s=t_{n-d}$.

The above construction obviously preserves the conditions for $f_{\mathrm{ref}}(t)$ and $g_{\mathrm{ref}}(t)$ for staying feasible at the points of $T_{n}$; i.e., we still have

$$
f_{\text {ref }}(t) \leq 0
$$

for every $t \in T_{n} \cap[\ell, s]$ and

$$
g_{\text {ref }}(t) \leq h(t)
$$

for every $\left.t \in T_{n} \cap[\ell, 1)\right)$.

Therefore, only the positive definiteness of $f_{\text {ref }}(t)$ and $g_{\text {ref }}(t)$ remains to be investigated. We remark that the new polynomials have obviously $f_{2 k}>0$ and $g_{2 k}>0$. Moreover, it follows from the construction that for every $i=0,1, \ldots, k$ we have $f_{\text {ref }}\left(\alpha_{i}\right) \geq 0$ and $g_{\text {ref }}\left(\alpha_{i}\right) \geq h\left(\alpha_{i}\right)$ with equality if and only if $\alpha_{i} \in T_{n}$. Thus the quadrature formula (30) implies that the new polynomials have $f_{0}>0$ and $g_{0}>0$. Furthermore, it also implies that

$$
\frac{f_{2 k}^{n, \ell, s}(1)}{f_{0}} \geq \frac{f_{\mathrm{ref}}(1)}{\left(f_{\mathrm{ref}}\right)_{0}}, \quad g_{0}-\frac{g_{2 k}^{n, \ell, M}(1)}{M} \leq\left(g_{\mathrm{ref}}\right)_{0}-\frac{g_{\mathrm{ref}}(1)}{M},
$$

so the bounds (34) and (35) are indeed improved as claimed above (provided the new polynomials are still feasible).

Numerical investigation of the remaining feasibility conditions $f_{i} \geq 0\left(g_{i} \geq 0\right)$ for $i=$ $1,2, \ldots, 2 k-1$ show that they are satisfied in numerous cases. Moreover, numerics lead us to the following conjecture concerning the relaxation of the linear programing over the discrete subset $[\ell, s] \cap T_{n}$ or $[\ell, 1] \cap T_{n}$ as introduced above.

Conjecture 8.1 For fixed $q \geq 3, n$, and $\ell$ there exists a constant $s(q, n, \ell)$ such that whenever $s \in[-1, s(q, n, \ell)) \cap T_{n}$ (that is large enough $d / n=(1-s) / 2$ ) the new polynomials $f_{\text {ref }}$ and $g_{\text {ref }}$ solve the relaxed linear programming in the context above

In other words, we conjecture that for most parameters the roots $\alpha_{1}, \alpha_{2}, \ldots, \alpha_{k-1}$ of the Levenshtein-type polynomial $f_{2 k}^{n, \ell, s}(t)$ are the best approximation of the optimal nodes for general linear programming. This implies significantly faster computation compared, for example, to the simplex method (see [5, 30]). More detailed investigation in this direction will be considered elsewhere.

\section{Upper energy bounds for codes with given cardinality and min- imum and maximum distance}

For given $q, n, M, s$, and $\ell$, denote by

$$
C_{n, q}(M, \ell, s):=\left\{C \subset F_{q}^{n}:|C|=M, s(C)=s, \ell(C)=\ell\right\}
$$


the set of codes in $F_{q}^{n}$ of cardinality $M \in\left[2, q^{n}\right]$, minimum distance $d=n(1-s) / 2$ and diameter $D=n(1-\ell) / 2$. In this section we derive a universal upper bound on the quantity

$$
\mathcal{U}_{h}(n, M, \ell, s):=\max \left\{E_{h}(C): C \in C_{n, q}(M, \ell, s)\right\},
$$

where $h$ is absolutely monotone.

The linear programming problem in this case can be formulated as follows

$$
\mathcal{U}_{h}(n, M, \ell, s) \leq \min _{p(t) \in \mathcal{Q}_{n, M, \ell, s}^{(h)}} M\left(p_{0} M-p(1)\right),
$$

with

$$
\begin{gathered}
p(t)=\sum_{i=0}^{n} p_{i} Q_{i}(t), \\
\mathcal{Q}_{n, M, \ell, s}^{(h)}:=\left\{p(t) \in F_{\leq}: p(t) \geq h(t), t \in[\ell, s]\right\},
\end{gathered}
$$

where $F_{\leq}:=\left\{p(t): p_{i} \leq 0, i=1,2, \ldots, n\right\}$.

We construct polynomials which belong to the set $\mathcal{Q}_{n, M, \ell, s}^{(h)}$ and therefore provide upper bounds for $\mathcal{U}_{h}(n, M, \ell, s)$ by (40). Let $s \in I_{k}^{(\ell)}, \alpha_{0}=\ell, \alpha_{1}, \ldots, \alpha_{k-1}, \alpha_{k}=s$ be the roots of $f_{2 k}^{n, \ell, s}(t)$ as above, and $\rho_{0}, \rho_{1}, \ldots, \rho_{k}$ are the corresponding weights from (30). Note that the parameters of the quadrature (30) no longer come with $M$ but with $s$ instead.

We consider

$$
p_{2 k}^{n, M, \ell, s}(t):=-\lambda f_{2 k}^{n, \ell, s}(t)+g_{L}(t)=\sum_{i=0}^{2 k} p_{i} Q_{i}(t),
$$

where $\lambda>0$ is a parameter (to be determined and optimized later) and

$$
g_{L}(t):=H\left(f_{2 k}^{n, \ell, s}(t) ; h(t)\right)
$$

is the Hermite interpolation polynomial to the function $h(t)$ that agrees with $h(t)$ exactly in the roots of the Levenshtein-type polynomial $f_{2 k}^{n, \ell, s}(t)$ (counted with their multiplicities).

Note that $\operatorname{deg}\left(g_{L}\right) \leq 2 k-1$ and therefore $\operatorname{deg}\left(p_{2 k}^{n, M, \ell, s}\right)=2 k$. Let

$$
f_{2 k}^{n, \ell, s}(t)=\sum_{i=0}^{2 k} f_{i} Q_{i}(t), \quad g_{L}(t)=\sum_{i=0}^{2 k-1} g_{i} Q_{i}(t)
$$

be the Krawtchouk expansions of $f_{2 k}^{n, \ell, s}(t)$ and $g_{L}(t)$, respectively.

The next theorem is the main result in this section.

Theorem 9.1 Let $n, q, k, \ell$, and $s$ be such that the conditions of Theorem 5.2 are fulfilled and let $C \in C_{n, q}(M, \ell, s)$. Then

$$
E_{h}(C) \leq \frac{p_{2 k}^{n, M, \ell, s}(1) M\left(M-L_{2 k}(n, \ell, s)\right)}{L_{2 k}(n, \ell, s)}+M^{2} \sum_{i=0}^{k} \rho_{i} h\left(\alpha_{i}\right)
$$


for every large enough $\lambda$. In particular,

$$
\begin{aligned}
& \mathcal{U}_{h}(n, M, \ell, s) \leq U_{2 k}(n, M, \ell, s) \\
= & \frac{p_{2 k}^{n, M, \ell, s}(1) M\left(M-L_{2 k}(n, \ell, s)\right)}{L_{2 k}(n, \ell, s)}+M^{2} \sum_{i=0}^{k} \rho_{i} h\left(\alpha_{i}\right),
\end{aligned}
$$

where $\lambda$ is chosen by

$$
\lambda:=\max \left\{\frac{g_{i}}{f_{i}}: 1 \leq i \leq 2 k-1\right\} .
$$

The bound (42) can be attained only by codes which have all their inner products in the roots of $f_{2 k}^{n, \ell, s}(t)$ and $p_{i} B_{i}^{\prime}=0$ for $i=1,2, \ldots, 2 k,\left(B_{0}^{\prime}, B_{1}^{\prime}, \ldots, B_{n}^{\prime}\right)$ is the MacWilliams transform of the attaining code.

Proof. Since $p_{i}=-\lambda f_{i}+g_{i}$ and $f_{i}>0$ for every $i=0,1, \ldots, 2 k$, it follows that large enough $\lambda>0$ will make $p_{i} \leq 0$ for every $1 \leq i \leq 2 k-1$. Adding the obvious $p_{2 k}<0$, we conclude that $p_{2 k}^{n, M, \ell, s}(t) \in F_{\leq}$.

Moreover, the absolute monotonicity of $h(t)$ and the interpolation conditions for $g_{L}(t)$ imply that $g_{L}(t) \geq h(t)$ for $t \in[\ell, s]$. Since $f_{2 k}^{n, \ell, s}(t) \leq 0$ for $t \in[\ell, s]$ and $p_{2 k}^{n, M, \ell, s}\left(\alpha_{i}\right)=g_{L}\left(\alpha_{i}\right)=h\left(\alpha_{i}\right)$ for every $i=0,1, \ldots, k$, it follows from (41) that $p(t) \geq h(t)$ for every $t \in[\ell, s]$ (whatever $\lambda>0$ is). Therefore $p_{2 k}^{n, M, \ell, s}(t) \in \mathcal{Q}_{n, M, \ell, s}^{(h)}$ for large enough $\lambda$ and it remains to compute the corresponding bound.

We first note that $L_{2 k}(n, \ell, s) \geq M$ follows from the monotonicity of the bound (34). Expressing $p_{0}$ by the quadrature formula (30) and using the interpolation conditions we obtain

$$
\begin{aligned}
& p_{0} M-p_{2 k}^{n, M, \ell, s}(1) \\
= & \left(\frac{M}{L_{m}(n, s)}-1\right) p_{2 k}^{n, M, \ell, s}(1)+M \sum_{i=0}^{k} \rho_{i} h\left(\alpha_{i}\right),
\end{aligned}
$$

whence we get (42) with $\lambda$ still to be optimized. The dependence of the right hand side of (42) on the parameter $\lambda$ comes from $p_{2 k}^{n, M, \ell, s}(1)$ only. Since $p_{2 k}^{n, M, \ell, s}(1)$ is linear and increasing with respect to $\lambda$, the best bound is obtained when $\lambda$ is chosen as in (43); i.e., when it is the smallest possible real number which satisfies all conditions $p_{i}=-\lambda f_{i}+g_{i} \leq 0, i=1,2, \ldots, 2 k-1$, simultaneously. Note that $\lambda>0$ by (43) since at least one of the ratios, $g_{2 k-1} / f_{2 k-1}$, is positive.

The description of necessary conditions for attaining codes is similar to that in Theorem 5.2 ,

Corollary 9.2 The energy of every code from $C_{n, q}(M, \ell, s)$ belongs to the interval $[L, U]$, where

$$
L:=M^{2} \sum_{i=0}^{k} \rho_{i}^{\prime} h\left(\alpha_{i}^{\prime}\right)
$$

(the parameters are determined by $M=L_{2 k}\left(n, \ell, s^{\prime}\right)$; i.e., by $M$ ) and

$$
U:=\frac{M\left(M-L_{2 k}(n, \ell, s) f(1)\right)}{L_{2 k}(n, \ell, s)}+M^{2} \sum_{i=0}^{k} \rho_{i} h\left(\alpha_{i}\right)
$$


(the parameters are determined by $f_{2 k}^{n, \ell, s}(t) ;$ i.e., by $\left.s\right)$, respectively.

A modification that adds $\ell$ to the interpolation nodes for $g_{L}(t)$ (so $\ell$ becomes a double node) works in a similar way as in Theorem 9.1 However, it is not difficult to prove that the bound produced is the same.

It is clear that the refining technique from Section 8 can be applied for improving the bounds (42) and, consequently, for shrinking the interval $[L, U]$ from Corollary 9.2 .

\section{Examples}

\subsection{System of bounds for $q=2$ and $\ell=-1+2 / n$}

We show as a typical example the mix of the Levenshtein bounds (see [26, Table 6.3]) and our Levenshtein-type bounds (34) for $\mathcal{A}_{2}(n, \ell, s)$, where

$$
\ell=t_{1}=-1+\frac{2}{n}=\frac{2-n}{n}
$$

is fixed (this $\ell$ corresponds to $D=n-1$, the second largest possible diameter). The fist four bounds (two Levenshtein bounds and our bounds for $k=1$ and $k=2$ ) are explicitely stated.

For $s \in[\ell,-1 / n]$ the first Levenshtein bound

$$
A_{2}\left(n, \frac{2-n}{n}, s\right) \leq \frac{s-1}{s}
$$

is valid. Our bound (34) for $k=1$

$$
A_{2}\left(n, \frac{2-n}{n}, s\right) \leq L_{2}\left(n, \frac{2-n}{n}, s\right)=\frac{2(1-s)(n-1)}{1-(n-2) s}
$$

is valid for

$$
s \in\left(t_{1,1}^{1,0}, t_{1,1}^{1, \ell}\right]=\left(-\frac{1}{n}, \frac{2}{n(n-3)}\right] .
$$

Then the next Levenshtein bound

$$
A_{2}\left(n, \frac{2-n}{n}, s\right) \leq \frac{(1-s) n(2+(n+1) s)}{1-n s^{2}}
$$

comes for

$$
s \in\left(t_{1,1}^{1,1}, t_{2,2}^{1,0}\right]=\left(0, \frac{\sqrt{n-1}-1}{n}\right] .
$$

Our bound (34) for $k=2$ is given by

$$
\begin{aligned}
& A_{2}\left(n, \frac{2-n}{n}, s\right) \leq L_{4}\left(n, \frac{2-n}{n}, s\right) \\
= & \frac{2(1-s) n(n-1)(9 s(n-4)+n-18)^{2}}{81 n A s^{3}+9 B s^{2}+C s+D},
\end{aligned}
$$


where

$$
\begin{aligned}
& A=n^{3}-9 n^{2}+25 n-18 \\
& B=29 n^{3}-207 n^{2}+351 n-162, \\
& C=n^{5}-38 n^{4}+369 n^{3}-675 n^{2}-648 n+972, \\
& D=-n^{4}+36 n^{3}-279 n^{2}+81 n+162 .
\end{aligned}
$$

It is valid for

$$
\begin{aligned}
& s \in\left(t_{2,2}^{1,0}, t_{2,2}^{1, \ell}\right] \\
= & \left(\frac{\sqrt{n-1}-1}{n}, \frac{2+\sqrt{n^{3}-12 n^{2}+41 n-26}}{n(n-5)}\right) .
\end{aligned}
$$

The even weight codes $C \subset F_{q}^{n}$, where $n=2 m+1$ is odd, attain the bound

$$
L_{2(m-1)}\left(2 m+1, \frac{2-n}{n}, \frac{n-4}{n}\right)=2^{2 m}
$$

and the corresponding bound (35).

\subsection{Cases $k=1$ and $k=2$}

As mentioned above, the case $k=1$ was considered by Helleseth, Klove and Levenshtein [21]. In our notations, their bound (see [21, Theorem 1]) is

$$
\mathcal{A}_{q}(n, \ell, s) \leq \frac{L}{L+4(q-1)(1-n)+2 n q(q-1)(s+\ell)},
$$

where $L=n q^{2}(1-s)(1-\ell)$.

Examples with $\ell=-1$ are covered by the Levenshtein bounds (see, for example, Table 1 in 25]). For $\ell>-1$ and $k=1$, we extract the following examples from [21].

For $n=6, q=2, \ell=-1 / 3(D=4), s=1 / 3(d=2)$ an explicit nonlinear code in [21. Example 1] has cardinality $M=16=L_{2}(6,-1 / 3,1 / 3)$. For $n=5, q=2, \ell=-3 / 5$ $(D=4), s=1 / 5(d=2)$ the binary [5,4,2] even weight code in [21, Example 2] has cardinality $M=16=L_{2}(5,-3 / 5,1 / 5)$. For $n=56, q=3, \ell=-17 / 28(D=45), s=-2 / 7(d=36)$ the Hill (ternary) projective cap (see [22]) has cardinality $M=729=L_{2}(56,-17 / 28,-2 / 7$ ) and for $n=78, q=4, \ell=-25 / 39(D=64), s=-17 / 39(d=56)$ the Hill (quaternary) projective cap (see [23]) has cardinality $M=729=L_{2}(56,-17 / 28,-2 / 7)$. All these codes have $h$-energies which attain (35) for the corresponding parameters and for every absolutely monotone $h$. In all cases the distance distributions of the attaining codes are easily computed by Theorem 6.2 (see Example 6.3).

The ovoids in $\operatorname{PG}(3, q)$ (see [13]) are codes $C \subset F_{q}^{n}$ with parameters

$$
n=q^{2}+1, d=q^{2}-q, D=q^{2},|C|=q^{4} .
$$


Thus we have

$$
s=\frac{1+2 q-q^{2}}{1+q^{2}}, \ell=\frac{1-q^{2}}{1+q^{2}}
$$

and the bound (34) is attained,

$$
|C|=M=q^{4}=L_{2}\left(q^{2}+1, \frac{1-q^{2}}{1+q^{2}}, \frac{1+2 q-q^{2}}{1+q^{2}}\right) .
$$

The distance distribution of these codes can be computed by the system (38) (for $k=1$; as in Example 6.3) ) and is given by

$$
\begin{gathered}
A_{\ell}=(q-1)\left(q^{2}+1\right)=n(q-1), \\
A_{s}=q(q-1)\left(q^{2}+1\right)=n d .
\end{gathered}
$$

Thus, in turn we find

$$
\begin{aligned}
& \rho_{0}=A_{\ell} \rho_{2}=\frac{n(q-1)}{q^{4}}, \\
& \rho_{1}=A_{s} \rho_{2}=\frac{n(q-1)}{q^{3}} .
\end{aligned}
$$

Then the energy of $C$ (attaining the bound (35) for every absolutely monotone $h$ ) can be computed as

$$
\begin{aligned}
& E_{h}(C)=M^{2}\left(\rho_{0} h(\ell)+\rho_{1} h(s)\right) \\
= & q^{4}\left(q^{2}+1\right)(q-1)\left(h\left(\frac{1-q^{2}}{1+q^{2}}\right)+h\left(\frac{1+2 q-q^{2}}{1+q^{2}}\right)\right) .
\end{aligned}
$$

Even more interesting example coming from [21] is given by an infinite series of codes constructed by Dodunekov, Helleseth, and Zinoviev [19]. For a prime power $q$ and positive integers $m$ and $2 \leq N \leq q^{m}+1$, the length and the cardinality of the codes from [19] are given by

$$
n=\frac{q^{m}-1}{q-1} N,|C|=q^{2 m} .
$$

Further, for these codes we have

$$
\begin{gathered}
s=-\frac{(N-2) q^{m}-2(N-1) q^{m-1}+N}{N\left(q^{m}-1\right)}, \\
\ell=-\frac{q^{m}-2 q^{m-1}+1}{q^{m}-1},
\end{gathered}
$$

corresponding to $d=(N-1) q^{m-1}$ and $D=N q^{m-1}$, respectively. For these $\ell$ and $s$, the condition $f_{1} \geq 0$ for the polynomial $f_{2}^{n, \ell, s}(t)$ is satisfied for $N \geq 1+\left(q^{m}-q\right) / 2$ (this corresponds to condition (16) from [21]). For such $N$ and with the above $n, \ell$ and $s$ we have

$$
|C|=q^{2 m}=L_{2}(n, \ell, s) .
$$


The distance distribution of these codes is computed by the system (38) for $k=1$ (as in Example 6.3). We have

$$
\begin{gathered}
A_{\ell}=q^{2 m}-1-\frac{q\left(q^{m}-1\right) N}{q-1}, \\
A_{s}=\frac{q\left(q^{m}-1\right) N}{q-1} .
\end{gathered}
$$

The $h$-energy is given by

$$
E_{h}(C)=q^{2 m}\left(A_{\ell} h(\ell)+A_{s} h(s)\right)
$$

and attains the bound (35) for every absolutely monotone $h$.

Examples with $k=2$ are already rare. In fact, there are many cases with integer $L_{4}(n, \ell, s)$ but most of them (among the checked) fail to produce integer distance distributions from Theoremddcodes. Two well known attaining codes are the projections of the binary Golay codes of lengths 23 and 22. Indeed, the first projection of the binary Golay code has parameters $n=23, \ell=-9 / 23$ (i.e., $D=16$ ), $s=7 / 23$ (i.e., $d=8$ ) and

$$
|C|=2^{11}=L_{4}\left(23,-\frac{9}{23}, \frac{7}{23}\right)
$$

and the second projection has parameters $n=22, \ell=-5 / 11$ (i.e., $D=16$ ), $s=3 / 11$ (i.e., $d=8$ ) and

$$
|C|=2^{10}=L_{4}\left(22,-\frac{5}{11}, \frac{3}{11}\right) .
$$

Acknowledgments. The first author was partially supported by the National Scientific Program "Information and Communication Technologies for a Single Digital Market in Science, Education and Security (ICTinSES)", financed by the Bulgarian Ministry of Education and Science. The second author was supported, in part, by the Simons Foundation under CGM \#282207. The work of the third and fourth author was supported, in part, by the U. S. National Science Foundation under grant DMS-1516400. The fifth authors was supported in part by Bulgarian NSF contract DN02/2-2016. A preliminary version of this paper with material from Sections 1-5 appeared in the Proceedings of IEEE ISIT2019.

\section{References}

[1] A. Ashikhmin, A. Barg, Binomial moments of the distance distribution: bounds and applications, IEEE Trans. Inform. Theory, 45, 1999, 438-452.

[2] A. Ashikhmin, A. Barg, S. Litsyn, A new upper bound on the reliability function of the Gaussian channel, IEEE Trans. Inform. Theory, 46, 2000, 1945-1961.

[3] A. Ashikhmin, A. Barg, S. Litsyn, Polynomial method in coding and information theory, arXiv:math/9910175, 1999.

[4] A. Ashikhmin, A. Barg, S. Litsyn, Estimates of the distance distribution of codes and designs, IEEE Trans. Inform. Theory, 47, 2001, 1050-1061. 
[5] A. Barg, D. Jaffe, Numerical results on the asymptotic rate of binary codes, Codes and Association Schemes (A. Barg and S. Litsyn, eds.), DIMACS series, vol. 56, AMS, Providence, R.I., 2001, 25-32.

[6] P. Boyvalenkov, D. Danev, On maximal codes in polynomial metric spaces, in Proc. AAECC12, Lecture Notes in Computer Science, Springer 1255 (1997) 29-38.

[7] P. Boyvalenkov, D. Danev, On linear programming bounds for codes in polynomial metric spaces, Probl. Peredachi Inf., 34(2), 1998, 16-31 (in Russian); English translation in Probl. Inform. Transm. 34(2), 1998, 108-120.

[8] P. Boyvalenkov, P. Dragnev, D. Hardin, E. Saff, M. Stoyanova, Universal lower bounds for potential energy of spherical codes, Constr. Approx. 44, 2016, 385-415.

[9] P. Boyvalenkov, P. Dragnev, D. Hardin, E. Saff, M. Stoyanova, Energy bounds for codes and designs in Hamming spaces, Designs, Codes and Cryptography, 82, 2017, 411-433.

[10] P. Boyvalenkov, P. Dragnev, D. Hardin, E. Saff, M. Stoyanova, Next levels universal bounds for spherical codes: the Levenshtein framework lifted, submitted.

[11] P. Boyvalenkov, T. Marinova, M. Stoyanova, Nonexistence of few binary orthogonal arrays, Discrete Applied Mathematics, 217(P2), 2017, 144-150.

[12] P. Boyvalenkov, H. Kulina, Investigation of binary orthogonal arrays via their distance distributions, Probl. Peredachi Inf., 49, 2013, 28-40 (in Russian); English translation in Probl. Inf. Transm., 49, 2013, 322-332.

[13] R. Calderbank, W. M. Kantor, The geometry of two-weight codes, Bull. London Math. Soc., 18, 1986, 97-122.

[14] H. Cohn, A. Kumar, Universally optimal distribution of points on spheres, J. Amer. Math. Soc. 20, 2007, 99-148.

[15] H. Cohn, Y.Zhao, Energy-minimizing error-correcting codes, IEEE Trans. Inform. Theory 60, 2014, 7442-7450.

[16] P. J. Davis, P. Rabinowitz, Methods of Numerical Integration, 2nd ed. Academic Press, New York, 1984.

[17] P.Delsarte, An Algebraic Approach to the Association Schemes in Coding Theory, Philips Res. Rep. Suppl. 10, 1973.

[18] P. Delsarte, V.I. Levenshtein, Association schemes and coding theory, Trans. Inform. Theory 44, 1998, 2477-2504.

[19] S. Dodunekov, T. Helleseth, V. Zinoviev, On q-ary Grey-Rankin bound and codes meeting this bound, In Proc. IEEE Internat. Symp. on Information Theory 2004, 2004, 528.

[20] G. Fazekas, V. I. Levenshtein, On the upper bounds for code distance and covering radius of designs in polynomial metric spaces, J. Comb. Theory A70, 1995, 267-288. 
[21] T. Helleseth, T. Kløve, V. I. Levenshtein, A bound for codes with given minimum and maximum distances, In Proc. IEEE ISIT 2006, Seattle, USA, July 9-14, 2006, 292-296.

[22] R. Hill, On the largest size cap in $S_{5,3}$, Rend. Arad. Naz. Lincei, vol. 54, 1973, 378-384.

[23] R. Hill, Caps and groups, in Arti dei Covegni Lincei, Colloquio Inrern. sulle Theorie Comhinatorie, Roma, 1973, vol. 17 (Acad. Naz. Lincei), 1976, 384-394.

[24] V. I. Levenshtein, Designs as maximum codes in polynomial metric spaces, Acta Appl. Math. 25, 1992, 1-82.

[25] V.I. Levenshtein, Krawtchouk polynomials and universal bounds for codes and designs in Hamming spaces, IEEE Trans. Infor. Theory 41, 1995, 1303-1321.

[26] V.I. Levenshtein, Universal bounds for codes and designs, Handbook of Coding Theory, V.S. Pless and W. C. Huffman, Eds., Elsevier, 1998, Ch. 6, 499-648.

[27] V.M. Sidel'nikov, On extremal polynomials used to estimate the size of codes, Probl. Inform. Transm. 16, 1980, 174-186.

[28] G. Szegó, Orthogonal polynomials, Amer. Math. Soc. Col. Publ., 23, Providence, RI, 1939.

[29] V. A. Yudin, Minimal potential energy of a point system of charges, Discret. Mat. 4, 115-121 (1992) (in Russian); English translation in Discr. Math. Appl. 3, 1993, 75-81.

[30] http://doc.sagemath.org/html/en/reference/coding/sage/coding/code_bounds.html. 